\title{
Hfq links translation repression to stress-induced mutagenesis in $E$. coli
}

\author{
Jiandong Chen and Susan Gottesman \\ Laboratory of Molecular Biology, Center for Cancer Research, National Cancer Institute, Bethesda, Maryland 20892, USA
}

\begin{abstract}
Mismatch repair (MMR) is a conserved mechanism exploited by cells to correct DNA replication errors both in growing cells and under nongrowing conditions. Hfq (host factor for RNA bacteriophage Q $\beta$ replication), a bacterial Lsm family RNA-binding protein, chaperones RNA-RNA interactions between regulatory small RNAs (sRNAs) and target messenger RNAs (mRNAs), leading to alterations of mRNA translation and/or stability. Hfq has been reported to post-transcriptionally repress the DNA MMR gene mutS in stationary phase, possibly limiting MMR to allow increased mutagenesis. Here we report that Hfq deploys dual mechanisms to control mutS expression. First, Hfq binds directly to an $(\mathrm{AAN})_{3}$ motif within the mutS $5^{\prime}$ untranslated region (UTR), repressing translation in the absence of sRNA partners both in vivo and in vitro. Second, Hfq acts in a canonical pathway, promoting base-pairing of ArcZ sRNA with the mutS leader to inhibit translation. Most importantly, using pathway-specific mutS chromosomal alleles that specifically abrogate either regulatory pathway or both, we demonstrate that tight control of MutS levels in stationary phase contributes to stress-induced mutagenesis. By interacting with the mutS leader, Hfq serves as a critical switch that modulates bacteria from high-fidelity DNA replication to stress-induced mutagenesis.
\end{abstract}

[Keywords: mismatch repair; noncoding small RNA; RNA chaperone; ARN motif; adaptive mutagenesis]

Supplemental material is available for this article.

Received May 25, 2017; revised version accepted July 17, 2017.

Mismatch repair (MMR) systems are conserved from bacteria through higher eukaryotes, and mutation in MMR genes leads to hypermutation (Kunkel and Erie 2005; Li 2008; Fukui 2010). While most mutations in the genome are neutral and even harmful, a small portion of mutations allow better adaptation to selective pressures and thus are beneficial for adaptation, diversity, and evolution (Denamur and Matic 2006). Therefore, cells may adjust mutation rates according to their environment to optimize their adaptability. In bacteria such as Escherichia coli, the idea that cells elevate mutation rates in response to stress for better survival through accumulating beneficial mutations has been extensively studied and debated (Denamur and Matic 2006; Foster 2007). In particular, analysis of molecular pathways for adaptive mutagenesis (also known as stress-induced mutagenesis), in which the frequency of a mutation increases as cells are incubated for long periods (days), suggests that it is a regulated complex process that involves the MMR system (Denamur and Matic 2006; Foster 2007). An essential component of MMR is MutS, which initiates the repair process by recognizing mismatched bases in the DNA duplex. In E. coli, the cellular level of MutS was depleted in stationary phase, dependent on the RNA chaperone Hfq (host fac-

Corresponding author: gottesms@helix.nih.gov

Article published online ahead of print. Article and publication date are online at http://www.genesdev.org/cgi/doi/10.1101/gad.302547.117. tor for RNA bacteriophage $\mathrm{Q} \beta$ replication) (Feng et al. 1996; Tsui et al. 1997).

Hfq, a bacterial homolog of the eukaryotic and archaeal Lsm family RNA-binding proteins (Schumacher et al. 2002; Sun et al. 2002), is involved in many aspects of bacterial RNA metabolism (Vogel and Luisi 2011; Sobrero and Valverde 2012). Best characterized as a chaperone for regulatory small RNAs (sRNA), Hfq binds both sRNA and its cognate messenger RNA (mRNA) to promote intermolecular base-pairing interactions, resulting in alterations of mRNA translation and/or stability (Updegrove et al. 2016).

Hfq forms a ring-shaped homohexamer with three RNA-binding surfaces (proximal, distal, and lateral) and a flexible C-terminal tail (Updegrove et al. 2016). The proximal face binds the oligo $U$ tract present at the $3^{\prime}$ end of the Rho-independent terminator of Hfq-dependent sRNAs, and this binding is essential for sRNA stability and function (Otaka et al. 2011). The distal face of $E$. coli Hfq binds A-R(A/G)-N (any nucleotide) triplet repeats found in many mRNA 5' untranslated regions (UTRs) (Link et al. 2009; Robinson et al. 2014; Tree et al. 2014). Moreover, some sRNAs bind both the proximal and distal face, and, in these cases, E. coli Hfq can also use its highly positively charged lateral face as an auxiliary binding site for mRNAs; this lateral (or rim) site has also been 
implicated in duplex formation and RNA exchange (Panja et al. 2013; Schu et al. 2015).

Dozens of sRNAs and hundreds of mRNAs, including many characterized targets of sRNA regulation, are enriched by Hfq immunoprecipitation (Zhang et al. 2013; Bilusic et al. 2014; Tree et al. 2014; Holmqvist et al. 2016; Melamed et al. 2016). mutS mRNA, encoding the MMR initiator MutS, was highly enriched by Hfq immunoprecipitation in E. coli (Zhang et al. 2013; Melamed et al. 2016), and its expression was repressed by Hfq through an undefined mechanism (Feng et al. 1996; Tsui et al. 1997), suggesting that Hfq-dependent sRNAs are likely involved. In this study, we explored the mechanism and biological significance of Hfq-mediated mutS repression in $E$. coli. We found that two sRNAs repress mutS expression: SdsR, previously reported (Gutierrez et al. 2013), and ArcZ. More interestingly, we determined that the major repression of mutS in stationary phase by Hfq is sRNA-independent via direct Hfq action. We further demonstrated the importance of this translation repression in enabling elevated mutagenesis in stationary-phase cells.

\section{Results}

ArcZ, an $H f q$-dependent sRNA, represses mutS expression through direct base-pairing interactions

In experiments to probe RNAs coimmunoprecipitated with Hfq in E. coli (Zhang et al. 2013), we observed a significant enrichment in the $\mathrm{Hfq}$ immunoprecipitation samples of dozens of known mRNA targets as well as additional mRNAs that have not been reported previously as targets. One highly enriched mRNA was muts (17-fold compared with total mRNA). Reinforcing the suggestion that mutS might be subject to regulation by $\mathrm{Hfq}$ and sRNAs, levels of the mutS transcript were modestly increased in strains carrying a mutant of Hfq unable to bind sRNAs (1.5-fold compared with wild type) (Zhang et al. 2013). These data suggested that Hfq-dependent sRNAs are likely to contribute to mutS repression, consistent with previous reports by Tsui et al. (1997) and Gutierrez et al. (2013). Many mRNAs are regulated by multiple sRNAs (Mandin and Guillier 2013; Mika and Hengge 2013), and, given the importance of MutS in bacterial physiology, we further examined muts regulation by Hfq and sRNAs.

To search for sRNA regulators of muts mRNA, we constructed an L-arabinose-inducible mutS-lacZ chromosomal translational fusion reporter (Fig. 1A) and screened for sRNA regulators in the reporter strain by overproduction of each of 27 individual sRNAs from an sRNA library (Mandin and Gottesman 2010). mutS-lacZ expression was determined by $\beta$-galactosidase activity assay in strainseach overexpressing a single sRNA-and compared with that of the vector plasmid control (Fig. 1B). Overproduction of ArcZ or SdsR down-regulated mutS-lacZ expression more than twofold. ChiX, an efficient Hfq titrator when overexpressed (Moon and Gottesman 2011; Ellis et al. 2015), increased mutS-lacZ expression by 1.8 -fold.

ArcZ sRNA was predicted by TargetRNA2 (Kery et al. 2014) to base-pair with mutS close to its ribosome-binding site (RBS) (Fig. 1C). To confirm the putative pairing, ArcZ mutations (ArcZ*) and compensatory muts mutations $\left(\right.$ mutS $\left.^{\text {ArcZ }}{ }^{*}\right)$, each of which disrupts but together restore this predicted pairing, were generated (Fig. 1C). Mutations in ArcZ completely abolished mutS-lacZ repression; the compensatory mutS ${ }^{\text {ArcZ }}$-lacZ fusion was largely resistant to wild-type ArcZ overexpression but was efficiently repressed by ArcZ* (Fig. 1D), demonstrating that ArcZ sRNA represses mutS expression through direct basepairing.

Interestingly, our sRNA library screen also identified SdsR as a post-transcriptional mutS repressor. SdsR has been reported previously to base pair with mutS deep
A
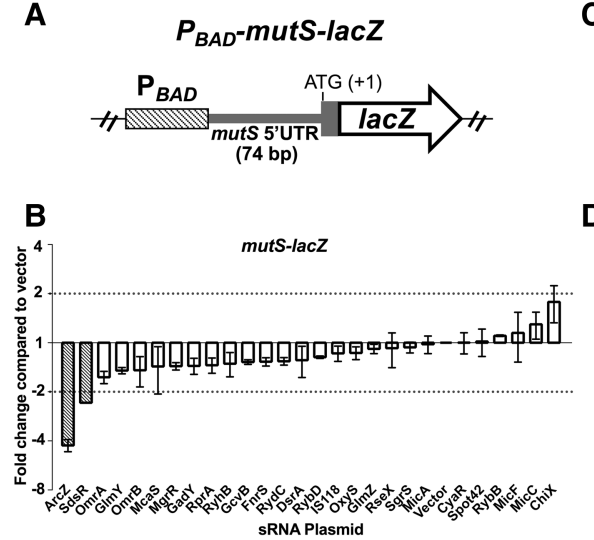

C

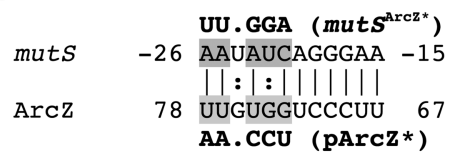

D

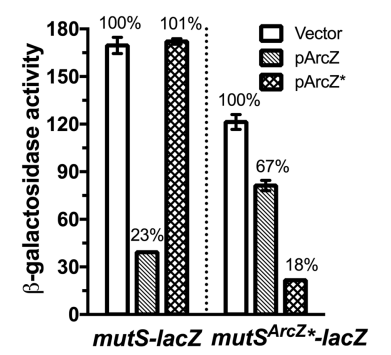

Figure 1. ArcZ sRNA represses mutS expression through direct base-pairing interactions. $(A)$ Schematic representation of mutS-lacZ chromosomal translational fusion. (B) Assays of mutS-lacZ reporter strain JC1006 in the presence of individual sRNAs overproduced from an $E$. coli sRNA library to identify sRNA regulators (shaded; greater than twofold changes compared with the vector control). The reporter strain harboring the sRNA-expressing plasmids was grown in Lennox broth (LB) ampicillin medium and induced with $0.01 \% \mathrm{~L}$-arabinose and $50 \mu \mathrm{M}$ IPTG for $6 \mathrm{~h}$ at $37^{\circ} \mathrm{C}$ before assaying $\beta$-galactosidase activity (in specific units). $\beta$-Galactosidase activities from each strain were normalized to that of the vector control pBRplac, set at 1 . The vector control strain gave a level of $\sim 15$ specific units. $(C)$ TargetRNA2-predicted RNA duplex formed between nucleotides -26 and -15 relative to the ATG of the mutS 5' UTR and nucleotides 67-78 of ArcZ sRNA, including G-U base pairs (colon). ArcZ numbering is based on the full-length transcript; ArcZ is rapidly processed at position 67. Point mutations that generate compensatory mutants ArcZ* and $m u t S^{A r c Z^{*}}$ are shown. $(D)$ sRNA and compensatory mRNA mutagenesis analyses for ArcZ regulation of $m u t S$ expression. Bacterial reporter strains (wild type: JC1006; mutS $^{\text {ArcZ* }}$ : JC1008) transformed with wild-type (pArcZ) or mutant (pArcZ*) ArcZ or the empty vector were induced with $0.01 \%$ arabinose and $50 \mu \mathrm{M} \mathrm{IPTG}$ for $6 \mathrm{~h}$ at $37^{\circ} \mathrm{C}$ before assay of $\beta$-galactosidase activities (in Miller units). Biological triplicates were assayed, and data are plotted as mean \pm SEM. 
within the coding sequence, based on in vitro annealing (Gutierrez et al. 2013), and this pairing region is absent in the translational fusion reporter used here, suggesting that SdsR can either base-pair with a different site in the mutS leader or indirectly affect mutS-lacZ translation. Overproduction of ArcZ or SdsR in a strain deleted for both endogenous sRNA genes down-regulated both reporter and endogenous MutS proteins similarly (Supplemental Fig. S1A,B), demonstrating that these two sRNAs can act independently of each other. ArcZ overproduction reduced the fusion and the endogenous protein by 6.3 fold, whereas SdsR down-regulated the fusion by 2.4-fold and the endogenous protein by 2.9-fold, further suggesting that the effects of both sRNAs are likely mediated primarily through the leader and initial translated region of muts; both the promoter and most of the coding sequence are missing in this reporter fusion.

A possible pairing region for SdsR close to the mutS RBS was predicted, and mutations in either SdsR or mutS alleviated SdsR repression (Supplemental Fig. S1C). However, the mut $S^{\text {SdsR* }^{*}}$ mutation did not restore repression by the compensatory SdsR* derivative. Northern blot analysis detected high-level expression of the SdsR* (Supplemental Fig. S1D). Possibly, high levels of SdsR* compete for Hfq. Therefore, while it is clear that SdsR can negatively regulate mutS expression within the leader, the precise mechanism or site of interaction remains to be proven.

$\mathrm{Hfq}$ is required for Arc $\mathrm{Z}$ accumulation and regulation of several targets (Papenfort et al. 2009; Mandin and Gottesman 2010; Soper et al. 2010), and thus we expected it to be essential for ArcZ-mediated mutS repression as well. To confirm this, we compared the effect of ArcZ overexpression on mutS-lacZ expression in the presence and absence of Hfq. ArcZ is rapidly processed from the primary transcript (120 nucleotides [nt]) to a functional sRNA (55 nt) by RNase E (Soper et al. 2010; Chao et al. 2017). As expected, the strong repression of mutS-lac $Z$ by overproduction of ArcZ was completely abolished in the $\Delta h f q$ strain; the base-pairing-defective ArcZ* was inert in both wild-type and $\Delta h f q$ strains (Supplemental Fig. S2A). Northern blot analysis (Supplemental Fig. S2B) showed that the short, but not the long, isoform of ArcZ was undetectable in the $\Delta h f q$ strains, consistent with previous findings that the short isoform of ArcZ was the major functional unit, and its accumulation is Hfq-dependent (Soper et al. 2010; Chao et al. 2017). Collectively, these results demonstrate that ArcZ sRNA, working together with the sRNA chaperone Hfq, represses mutS expression through direct base-pairing interactions. Notably, the expression level of the mutS-lacZ fusion was high in the absence of Hfa (Supplemental Fig. S2A), and this is further explored below.

\section{Endogenous ArcZ sRNA is sufficient to down-regulate mutS expression in stationary phase}

To determine whether endogenous ArcZ and SdsR repress mutS expression, we compared mutS expression in wildtype cells and cells deleted for arc $Z$, $s d s R$, or both sRNAs for chromosomal mutS-lacZ reporter expression and endogenous MutS protein levels (Fig. 2A,B). Deletion of ArcZ modestly increased mutS-lacZ expression and MutS protein levels in stationary phase $(1.5$-fold $[P<$ $0.01]$ and 2.4-fold $[P<0.05]$, respectively). In contrast, deletion of SdsR had a nonsignificant effect on reporter expression (1.1-fold) but did lead to a moderate increase (2.2-fold $[P<0.05])$ in MutS protein levels. Such discrepancy between reporter fusion and endogenous proteins could be attributed to higher expression of the fusion. It is also possible that the previously reported SdsR-pairing site $/ \mathrm{Gu}$ tierrez et al. 2013) contributes to MutS repression of the endogenous protein. The arc $Z s d s R$ double deletion did not significantly increase either the fusion or protein levels compared with the deletion of $\operatorname{arcZ}$ (Fig. 2A,B). ArcZ is expected to indirectly affect $s d s R$ transcription through stimulation of rpoS translation; SdsR is transcribed by RpoS (Supplemental Fig. S3A; Frohlich et al. 2012). Indeed, deletion of ArcZ significantly decreased endogenous levels of SdsR (Supplemental Fig. S3B). Therefore, ArcZ
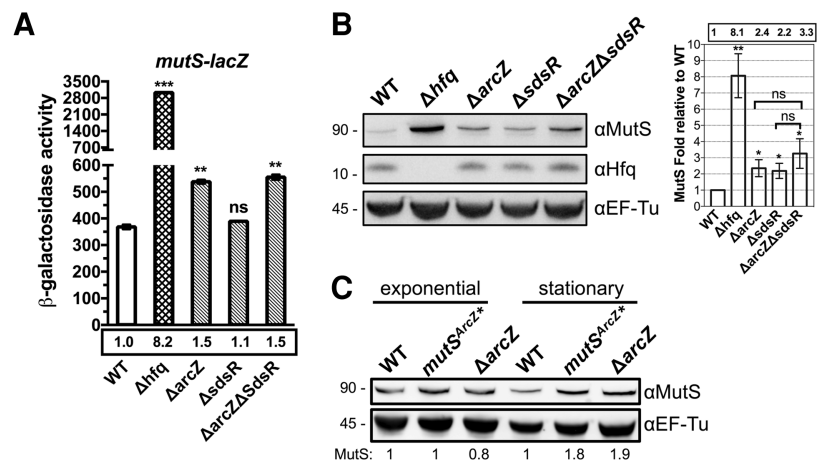

Figure 2. Endogenous ArcZ sRNA is sufficient to repress mutS expression in the stationary phase of bacterial growth. (A) $\beta$-Galactosidase activity assay to monitor mutS-lacZ expression in wild-type (JC1060) and isogenic mutant cells with deletion of hfq (JC1072), arcZ (JC1061), sdsR (JC1071), or arcZ sdsR (JC1073). Note that in these strains, the promoter for the mutSlacZ translational fusion is a constitutive synthetic $\mathrm{CP} 12 \mathrm{~b}$ promoter (Jensen and Hammer 1998) rather than the $P_{B A D}$ promoter used in Figure 1. Bacterial cells were grown in LB for $16 \mathrm{~h}$ at $37^{\circ} \mathrm{C}$ before assay of $\beta$-galactosidase activity (in Miller units). Biological triplicates were assayed, and data are plotted as mean \pm SEM. $(B)$ Western blot analysis of whole-cell lysates from wild type (NM525) and isogenic mutants with deletion of $h f q$ (JC1090), $\operatorname{arcZ}$ (JC1042), sdsR (JC1021), or arcZ sdsR (JC1069). Bacterial cells were grown in LB for $16 \mathrm{~h}$ at $37^{\circ} \mathrm{C}$ before Western blot analysis. Experiments were repeated four times; a representative blot is shown at the left, and MutS quantification, first normalized to the protein loading control EF-Tu and then to the normalized value of MutS for wild type, is at the right. Mean \pm SEM. (C) Western blot analysis of whole-cell lysates from wild-type (JC1081) and mutS $^{\text {ArcZ* }^{*}}$ (JC1083, carrying mutations of ArcZ base-pairing sequence in the mutS $5^{\prime}$ UTR) or $\triangle a r c Z$ sRNA (JC1042) cells. Bacteria grown in $\mathrm{LB}$ were collected at $\mathrm{OD}_{600}$ of 0.7 (exponential) and 4.5 (stationary), respectively. Representative blots from one of two experiments are shown. Relative MutS levels were calculated, with the wild-type sample for each group (exponential or stationary) set to 1 . Unpaired Student's $t$-test was used to calculate statistical significance. (ns) Not significant, $P>0.05 ;\left({ }^{*}\right) P<0.05$; $(* *) P<0.01 ;\left(^{* * *}\right) P<0.001$. 
likely represses mutS both directly by pairing and indirectly by stimulating the synthesis of SdsR, explaining the lack of additivity for the $\operatorname{arc} Z s d s R$ double deletion.

We further engineered the mutS compensatory mutant $\left(\right.$ mutS $\left.^{\text {ArcZ* }}\right)$ used in the fusion (Fig. 1D) into the native mutS leader to determine the effects of ArcZ direct pairing on endogenous mutS expression. Both mutS ${ }^{\text {ArcZ }}$ and $\triangle a r c Z$ increased MutS protein to similar extents (1.8fold and 1.9-fold, respectively) (Fig. 2C), compared with wild type in stationary phase, when ArcZ expression was high (Supplemental Fig. S3C). In contrast, such increases were not observed in exponentially growing cells (Fig. 2C) when ArcZ levels were low (Supplemental Fig. S3C). The observation that mutation of the ArcZ-pairing site in mut $S$ is almost as effective as deletion of $\operatorname{arc} Z$ in derepressing MutS levels also suggests that ArcZ's other roles-for instance, in stimulating synthesis of RpoS and thus SdsR (Supplemental Fig. S3) - are relatively minor under these conditions. Taken together, our data demonstrate that endogenous ArcZ is sufficient to down-regulate mutS expression in stationary phase in a sequence-specific manner.

\section{$H f q$ represses muts beyond $s R N A s$}

Our results showed a modest repression from endogenous ArcZ and SdsR on mutS expression (Fig. 2A,B), which is significantly less than the increase in an $h f q$ insertion mutant described previously by Tsui et al. (1997). Indeed, deletion of Hfq had a significantly greater impact on mutS expression than deleting either or both sRNAs (Fig. 2A,B), increasing the mutS-lacZ fusion and MutS protein levels by eightfold in the $\Delta h f q$ strain. These data suggested that other Hfq-dependent regulatory pathways also contribute to mutS repression. Such a pathway could involve other sRNAs not included in our sRNA library (Fig. 1B) or possibly other Hfq-dependent but sRNA-independent mechanisms.

The Hfq proximal face is used for sRNA binding and stabilization, and the distal face is used preferentially for mRNA association (Updegrove et al. 2016). We reasoned that if other sRNA regulators were responsible for the remaining mutS repression, then mutations in the proximal face of Hfq would phenocopy the $h f q$ deletion in terms of mutS expression. Therefore, we compared mutS expression in cells carrying chromosomal mutations in the native $h f q$ locus that disrupt the function of different Hfq faces (Fig. 3A,B; Zhang et al. 2013). The proximal face mutation (Q8A), which destabilizes Hfq-dependent sRNAs (Zhang et al. 2013; Schu et al. 2015), only modestly increased mutS expression (Fig. 3A,B), to a level comparable with that seen in the absence of ArcZ and SdsR sRNAs (see Fig. 2, which uses an independent set of wild-type and mutant strains) even though the Hfq Q8A protein was poorly detected by Western blot (Fig. 3B), possibly reflecting loss of epitopes important for antibody recognition. Mutation in the lateral face (R16A) showed an effect on fusion expression similar to that of Q8A and no increase of MutS protein levels (Fig. 3A,B). As expected, overexpression of ArcZ sRNA in the Q8A proximal face

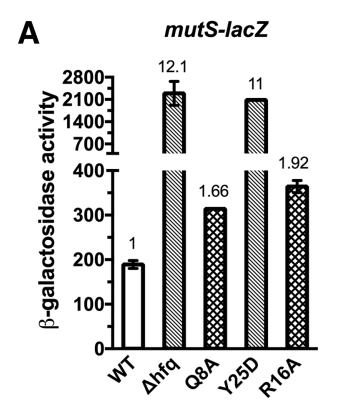

B

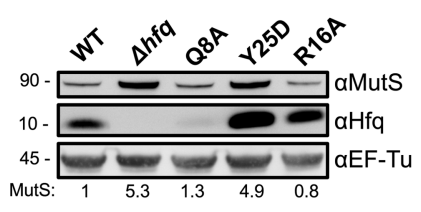

Figure 3. The canonical mRNA-binding distal face of Hfq is essential for mutS repression, but the sRNA-binding proximal face is not. (A) $\beta$-Galactosidase activity assays to determine mut $S$-lac $Z$ expression in isogenic strains carrying various $h f q$ alleles in the native chromosomal locus (wild type: JC1103; $\Delta h f q$ : JC1104; Q8A: JC1105; Y25D: JC1106; R16A: JC1107), where Q8A is an Hfq proximal face mutant, Y25D is a distal face mutant, and $\mathrm{R} 16 \mathrm{~A}$ is a lateral face mutant. These strains were generated by moving the kan-Cp12b-mutS-lacZ reporter into various Hfq allele strains reported previously (Zhang et al. 2013) and thus are similar to but independently derived from those used in Figure 2 (Supplemental Table S1). Bacterial cells were grown in LB for $6 \mathrm{~h}$ at $37^{\circ} \mathrm{C}$ before assaying for $\beta$-galactosidase activity (in Miller units). Biological triplicates were assayed for $\beta$-galactosidase activities, and data are plotted as mean \pm SEM. $(B)$ Western blot analysis of whole-cell lysates from the same set of strains as $A$. Bacterial cells were harvested at $\mathrm{OD}_{600}$ of $\sim 3.0$. For Western blot, a representative figure from biological duplicates is shown, and relative MutS levels are shown.

mutant did not repress mutS-lacZ expression; in the R16A lateral face mutant, repression was significantly reduced (Supplemental Fig. S2A). Both mutant $h f q$ alleles reduced or eliminated processed ArcZ sRNA (Supplemental Fig. S2B).

Strikingly, mutation in the distal face (Y25D), which binds to ARN motifs in mRNA targets (Link et al. 2009; Tree et al. 2014), significantly increased mutS expression for both the fusion and the endogenous protein to a level similar to that in the $h f q$ null strain (Fig. 3A,B). Interestingly, ArcZ sRNA down-regulated mutS-lacZ expression in the Y25D distal face mutant almost as well as in the wild-type strain (sixfold vs. fourfold) (Supplemental Fig. S2A), indicating that the distal face of Hfq is not essential for ArcZ-mediated mutS repression, at least under conditions of ArcZ overexpression. Taken together, the requirement of the distal face but not the proximal face of Hfq for mutS repression strongly suggests that the major mutS repression by Hfq is independent of sRNAs, as Hfq-dependent sRNAs are destabilized in the proximal face (Q8A) mutant (Zhang et al. 2013; Schu et al. 2015).

\section{An $(A A N)_{3}$ motif in the mutS $5^{\prime}$ UTR is essential for $\mathrm{H} f$-mediated mutS repression in vivo}

The distal face of Hfq binds to A tracts or (ARN) $\left.\right|_{n(n=3-6)}$ motifs frequently present in mRNA 5' UTRs (Link et al. 2009), with a preference for $(\mathrm{AAN})_{\mathrm{n}}$ repeats (Robinson et al. 2014). Such binding can rearrange the mRNA 
secondary structure and modulate the efficiency of mRNA translation (Geissmann and Touati 2004; Sonnleitner and Blasi 2014; Ellis et al. 2015). Given the critical role of the Hfq distal face for mutS repression, we searched for Hfq distal-face-binding elements (ARN repeats) in the 104-nt-long mutS leader and initial translated region (74nt-long $5^{\prime}$ UTR plus the first 10 codons of mutS). The Find Individual Motif Occurrences (FIMO) algorithm (Grant et al. 2011) identified two perfect $(\mathrm{ARN})_{3}$ repeats (ARN1 from -50 to -42 and ARN3 from +9 to +17 ) and one $(\mathrm{ARN})_{3}$ repeat with one nucleotide mismatch (ARN2 from -26 to -18), which overlaps the ArcZ-pairing sequence (Fig. 4A) and was mutated in the muts ${ }^{\text {ArcZ }}{ }^{*}$ mutant (Fig. 1D). There is a modest decrease in expression of mutS when ARN2 is disrupted in the mutS ${ }^{\text {ArcZ* }}$ mutant, and the mut $S^{\text {ArcZ }^{*}}$ is repressed well by the complementary ArcZ* sRNA (Fig. 1D), suggesting that the ARN2 motif is not needed for repression by Hfq or ArcZ. Mutation of ARN1 (ARN1*) increased mutS-lacZ expression by 5.8fold compared with that of wild type (Fig. 4B, cf. lanes 3 and 1), indicating that the ARN1 motif has a strong repressive effect on mutS expression. More importantly, deletion of $h f q$ in the ARN1* background only slightly increased mutS-lacZ expression (1.4-fold) compared with that of the wild-type $h f q$ ARN1* control (Fig. 4B, cf. lanes 4 and 3) and comparable with the effect of deleting arcZ (Fig. 2). Therefore, the ARN1 motif is required for Hfq-mediated muts repression in vivo. Mutation of ARN3

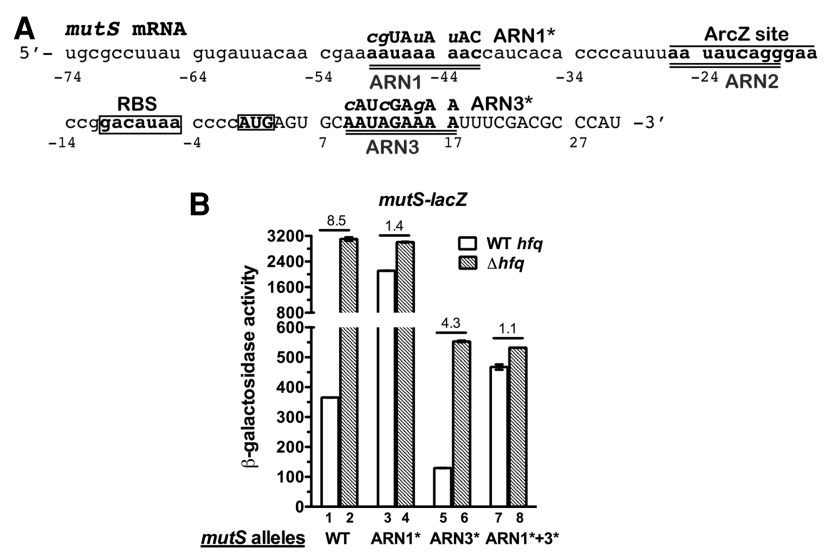

Figure 4. The ARN1 motif in the mutS $5^{\prime}$ UTR is essential for Hfq-mediated mutS repression in vivo. (A) Nucleotide sequence of $E$. coli mutS leader from the transcription start site $(-74)$ to $+30 \mathrm{nt}$ in the coding sequence. Sequence elements are highlighted in bold, with a single bar above the ArcZ base-pairing site, boxes around the RBS and the start codon (AUG), and double underline below the ARN sites. The ARN1 and ARN3 mutant sequences are shown with the mutated nucleotides in italic lowercase above. $(B)$ $\beta$-Galactosidase activity assays to determine mutS-lacZ expression in cells containing different mutS ARN alleles in the presence or absence of Hfq. Bacterial cells (wild type: JC1060; wildtype $\Delta h f q$ : JC1072; ARN1*: JC1111; ARN1* $\Delta h f q$ : JC1116; ARN3*: JC1112; ARN3* $\Delta h f q$ : JC1114; ARN1*+3*: JC1113; ARN1* $3^{*} \Delta h f q$ : JC1115) were grown in LB for $6 \mathrm{~h}$ at $37^{\circ} \mathrm{C}$ before assaying for $\beta$-galactosidase activity (in Miller units). Biological triplicates were assayed, and data are plotted as mean \pm SEM.
$(\mathrm{ARN} 3 *)$, changing the initial translated region of muts, decreased rather than increased mutS-lacZ expression (Fig. 4B, cf. lanes 5 and 1), presumably by affecting the initial codon usage in the fusion. Deletion of $h f q$ in the ARN3* background led to a significant increase (4.3-fold) of mutS-lacZ expression (Fig. 4B, cf. lanes 6 and 5), suggesting that ARN3 is not required for Hfq-mediated repression of mutS. An ARN1 and ARN3 double mutant (ARN1* + 3*) expressed mutS-lacZ at a level comparable with that of ARN3* in an $h f q$ mutant, and deletion of $h f q$ had very little additional effect (Fig. 4B, cf. lanes 7,8 and 6), further supporting the importance of the ARN1 motif in Hfq-mediated mutS repression. Collectively, these results support a model in which $\mathrm{Hfq}$ binds via its distal face to the ARN1 site in the muts 5' UTR, leading to mutS repression.

When Hfq catalyzes sRNA-mediated gene regulation, Hfq binds both the sRNA and the mRNA target using different faces (Schu et al. 2015). We tested whether the ARN1 or ARN3 site was required for ArcZ to repress mutS expression by overexpressing ArcZ in cells carrying the ARN1*, ARN3*, or ARN1* + ARN3* mutS-lacZ fusions (Supplemental Fig. S4B). In all of these mutS mutants, ArcZ repression was retained. These data were consistent with the observation that ArcZ efficiently repressed mutS-lacZ in the Hfq distal face mutant (Supplemental Fig. S2A), suggesting that ArcZ-mediated mutS repression requires different $\mathrm{Hfq}$ faces (proximal and lateral) and may use a different Hfq-binding site in mutS.

Similar experiments were performed to investigate the cis and trans elements required for SdsR-mediated mutS repression (Supplemental Fig. S4D,E). As expected, the Hfq proximal face mutation (Q8A) abolished SdsR repression. However, unlike ArcZ (Supplemental Fig. S2A), the distal face mutation (Y25D) but not the lateral face mutant (R16A) significantly reduced repression (Supplemental Fig. S4D). Consistent with the requirement for the distal face of Hfq, SdsR repression was partially affected in mutants containing ARN1 mutations (ARN1* and ARN1* $+3^{*}$ ) (Supplemental Fig. S4E). These data suggest that SdsR-mediated mutS repression uses the Hfq distal face, and, most likely, the mutS ARN1 motif contributes to $\mathrm{SdsR}$ repression.

\section{The ARN1 motif in the mutS 5' UTR is important for efficient $H f q$ binding and repression in vitro}

Our data suggest that Hfq-mediated, sRNA-independent mutS repression is the major repression pathway in $E$. coli (Fig. 2). We further examined this regulation in vitro through electrophoretic mobility shift assays (EMSAs), in vitro translation assays, and in vitro structure probing and footprinting analysis. EMSA analyses with different mutS alleles showed Hfq tightly bound to wild type or the ARN3* mutS leader, with apparent binding affinities $\left(K_{\mathrm{d}}\right)$ of $\sim 13$ and $19 \mathrm{nM}$, respectively. Mutating the ARN1 motif $(A R N 1 *)$ decreased the binding affinity by approximately threefold (Fig. 5A,B), demonstrating the importance of the ARN1 motif for efficient Hfq binding in vitro. Notably, the ARN1* mutation also decreased 
A

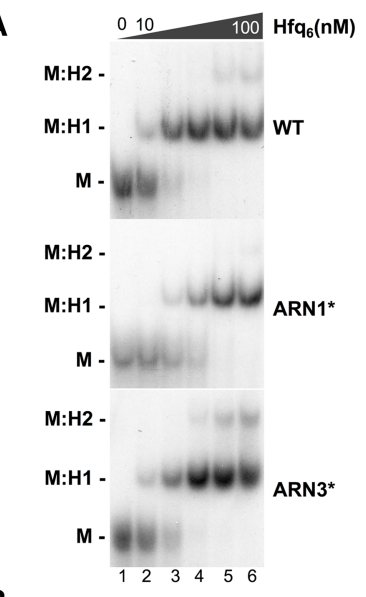

B
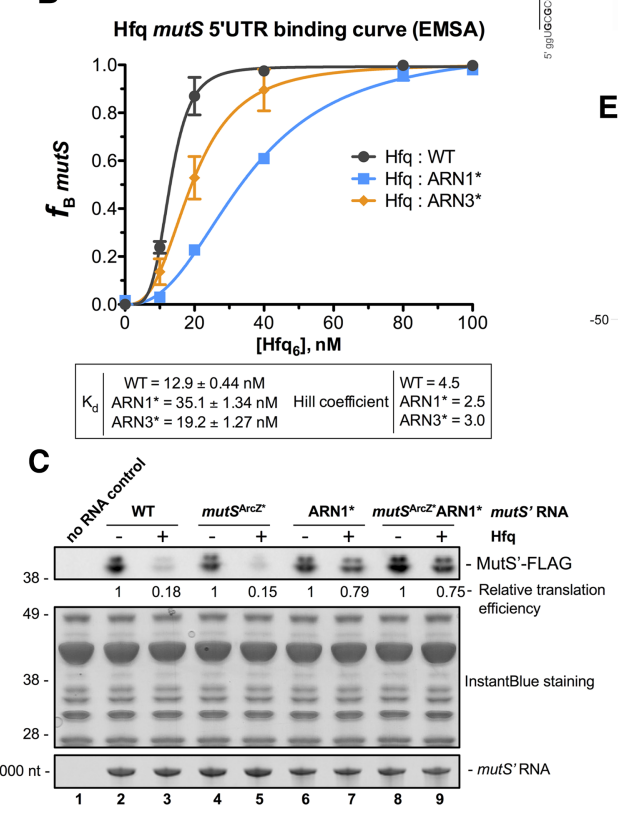
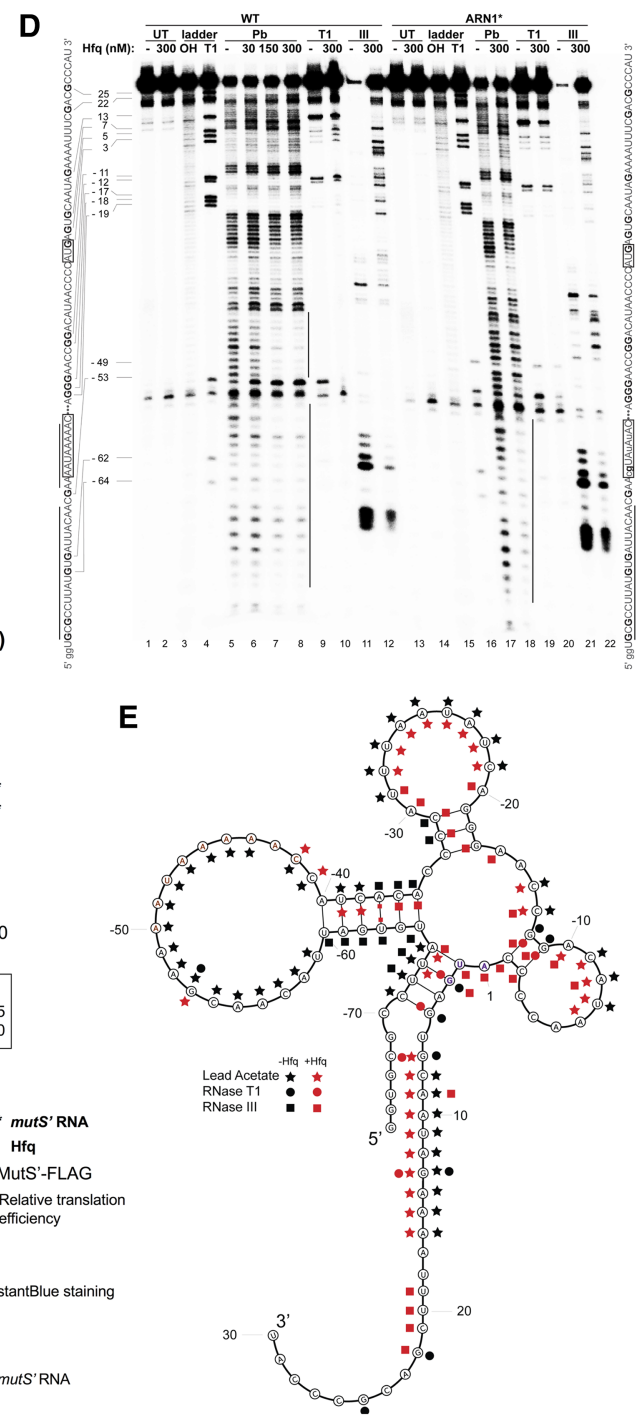

Figure 5. The ARN1 motif in the mutS $5^{\prime}$ UTR is important for efficient Hfq binding and translation inhibition in vitro. $(A)$ EMSA to measure the apparent equilibrium dissociation constants using purified $\mathrm{Hfq}$ and in vitro transcribed mutS (ARN) alleles. Purified $\mathrm{Hfq}_{6}$ final concentrations of 0,10 $20,40,80$, and $100 \mathrm{nM}$ were incubated with $1 \mathrm{nM} 5^{\prime}$ end ${ }^{32}$ P-labeled mutS mRNAs. Hfq binds to the mutS leader, first forming a smaller ribonucleoprotein (RNP) complex (M:H1) and then forming a larger complex (M:H2) in the presence of excess Hfq. (B) Quantification of Hfq-binding affinities to different mutS alleles in A. ImageStudio software was used to quantify the intensity of free labeled RNA and RNP bands, and then RNP signals were normalized to total signal in each lane and plotted using Prism 5 software (Graphpad) as the fraction of RNA bound $\left(f_{B}\right.$ mut $\left.S\right)$ versus the concentration of $\mathrm{Hfq}_{6}$. Data from two independent experiments are plotted as mean \pm SEM. The $K_{\mathrm{d}}$ and Hill coefficient were calculated using Graphpad. (C) In vitro translation of mutS'Flag mRNAs in the presence or absence of Hfq. mutS'-Flag mRNAs, including wild type, the ArcZ-pairing mutant $\left(\right.$ mut $\left.^{\text {ArcZ }}{ }^{*}\right)$, ARN1*, and the mutS ${ }^{\text {ArcZ }}$ ARN1* double mutant, were in vitro transcribed, and equimolar amounts $(2.5 \mathrm{pmol})$ of purified RNA and $\mathrm{Hfq}_{6}$ were mixed and subjected to in vitro translation using the PURExpress kit. The translated MutS'-Flag proteins were detected using anti-Flag antibodies. Total proteins from reactions were stained with InstantBlue, and mutS RNAs extracted from in vitro translation reactions were resolved on $6 \%$ TBE-urea gel and stained with ethidium bromide. MutS'-Flag protein signals were quantified by ImageStudio software and normalized to corresponding RNA amounts to determine the translation efficiencies. Relative translation inhibition for each mut $S$ allele in the presence of Hfq was determined by normalizing the translation efficiency to that in the absence of Hfq. $(D)$ In vitro footprinting and structure probing of $5^{\prime}$ end-labeled mut $S$ wild-type and ARN1* mRNAs ( $30 \mathrm{nM}$ in $10 \mu \mathrm{L}$; total $0.3 \mathrm{pmol}$ ) by RNase T1, RNase III, or lead acetate (Pb) cleavage in the presence (concentrations as indicated) or absence of Hfq. The Hfq footprints on mutS mRNA are highlighted by vertical bars and underlined in the wild-type and ARN1* sequences, shown at the left and right, respectively. ARN1 sequences and AUG are in boxes, and ARN1* mutant changes are shown with lowercase letters. G residues are shown in bold. A 21-nt sequence between nucleotides -41 and -21 (CATCACACCCCATTTAATATC), not affected by Hfq, is shown as an ellipsis to allow the rest of the sequence to be readable. (UT) Untreated $5^{\prime}$ end-labeled mutS RNA in the presence $(300 \mathrm{nM})$ or absence $(-)$ of Hfa proteins. Lanes OH and T1 show alkaline or RNase T1 ladders of denatured mutS RNA. (E) Secondary structure of wild-type mutS leader based on top hit for Nucleic Acid Package (NUPACK) (Zadeh et al. 2011) and RNA Structure Web server (http://rna.urmc.rochester.edu/RNAstructureWeb/Servers/ Predict1/Predict1.html) predictions; this structure also agrees well with chemical and enzymatic (RNase T1, RNase III, and lead) structure probing ( shown in $D$ ). An alternative structure in the presence of Hfq is shown in Supplemental Figure S9.

cooperative binding compared with wild type, reducing the Hill coefficient from 4.5 in wild type to 2.5 in ARN1*.

To determine the outcome of reduced Hfq binding, we carried out in vitro translation assays using purified Hfq protein and in vitro transcribed mutS'-Flag RNA variants (described in Supplemental Fig. S5A). In the presence of increasing amounts of $\mathrm{Hfq}$, we observed a dose-dependent inhibition of muts translation, with a fourfold reduction in MutS'-Flag production in the presence of equimolar amounts of Hfq hexamer and wild-type mutS mRNA (Supplemental Fig. S5B). Thus, Hfq can effectively inhibit mutS translation in the absence of sRNA and endonucleases. Most importantly, this Hfq-mediated translation inhibition was dependent on the ARN1 site. Mutating the ARN1 motif (ARN1* or mutS ArcZ* $^{*}$ ARN1*) restored robust translation in the presence of Hfq compared with the wildtype ARN1 alleles (wild-type and mutS ArcZ*) (Fig. 5C, cf. lanes 7,9 and 3,5). Neither the mutS $S^{\text {ArcZ }^{*}}$ nor the ARN1* 
mutation itself substantially affected mutS translation in the absence of Hfq (Fig. 5C, cf. lanes 2 and 4,6,8), and similar levels of total RNA and proteins were present in each reaction (Fig. 5C, bottom). Based on these results, we conclude that Hfq can directly inhibit mutS translation in vitro dependent on the ARN1 motif and largely independent of RNA degradation.

We further explored the basis for Hfq-mediated direct repression on muts by performing in vitro structure probing and footprinting analyses using wild-type and ARN1* muts leaders with and without purified Hfq (Fig. 5D). The ARN1 site is far ( 40 nt) upstream of the RBS, and a direct blockade of ribosome loading is unlikely. Instead, we favor a model in which Hfq binding at the ARN1 motif restructures the mutS leader, leading to the observed repression. Our results from chemical (lead acetate) and enzymatic (RNase T1 and RNase III) cleavage agreed well with the Nucleic Acid Package (NUPACK) prediction (Zadeh et al. 2011) for the secondary structure of the mutS leader in the absence of $\mathrm{Hfq}$ and are summarized in Figure 5E. The mutS ARN1 motif (from -50 to -42 ) is singlestranded in the absence of Hfq and was strongly protected by Hfq from lead $(\mathrm{Pb})$ cleavage in the wild-type mutS leader (Fig. 5D [cf. lanes 7,8 and 5], E [cf. red star \{with Hfq\} and black star $\{$ no Hfa\} $]$ ); this protection was completely lost in the ARN1* RNA (Fig. 5D, cf. lanes 18 and 17). Besides the ARN1 site, an upstream region $(-72$ to -55$)$ was also protected by Hfq from lead cleavage in both the wild type and ARN1* mutS leader. Therefore, this U-rich $5^{\prime}$ region is an ARN1-independent Hfq-binding site that likely contributes to the remaining binding of $\mathrm{Hfq}$ to the ARN1* muts leader seen in Figure 5A. Deletion of this site $\left(\Delta 5^{\prime}\right)$ (Supplemental Fig. S4A) increased mutS-lacZ expression by 1.7-fold (Supplemental Fig. S4C, cf. lanes 9 and 1) and decreased Hfq repression from 8.5-fold to 2.6-fold (Supplemental Fig. S4C, cf. lanes 1,2 and 9,10), suggesting that it also contributes to Hfq-mediated mutS repression. Notably, this site was not required for ArcZ-mediated mutS repression (Supplemental Fig. S4B). Strikingly, addition of Hfq drastically changed the pattern of cleavage by RNase III, which cleaves dsRNA, decreasing double-stranded regions formed at the $5^{\prime}$ end of the muts leader and increasing double-stranded structures at the $3^{\prime}$ end of the RNA (Fig. 5D [lanes 11,12], E [cf. red squares and black squares]). In the ARN1* mutant, the changes from RNase III cleavage upon Hfq addition were less dramatic, and a new region of RNase III cleavage was seen at the ARN1* site (Fig. 5D, cf. lanes 21,22 and 11,12). Collectively, these data support the ARN1 motif as a major site of Hfq binding, and binding to this site by Hfq remodels the mutS leader in a manner that leads to mutS repression.

\section{Hfq-mediated mutS repression contributes to bacterial mutagenesis}

Our results demonstrate that Hfq deploys dual mechanisms to control mutS expression: repression via ArcZ and SdsR sRNA and direct repression by Hfq. Here we further explored the contributions of both repression pathways to bacterial mutagenesis. Deletion of mutS in $E$. coli significantly increases mutation rates (LeClerc et al. 1996; Bjedov et al. 2003), with a >50-fold increase compared with wild type in the frequencies of spontaneous mutagenesis to rifampicin resistance and reversion of a lac ochre mutation to $\mathrm{Lac}^{+}$(Supplemental Fig. S6A,B). MMR proteins have also been implicated in bacterial adaptive or stress-induced mutagenesis (Harris et al. 1999; Bjedov et al. 2003; Denamur and Matic 2006; Foster 2007). Using a classic assay for stress-induced mutagenesis that measures correction of a +1 frameshift mutation in lacI-lacZ fusion by growth on lactose minimal agar over many days (Cairns and Foster 1991), we observed that the level of $\mathrm{Lac}^{+}$colonies over $6 \mathrm{~d}$ increased by $>30$ fold in $\Delta$ muts compared with wild type (Supplemental Fig. S7B).

MutS levels are high in rapidly growing E. coli cells and decrease significantly in stationary phase (Supplemental Fig. S8A; Feng et al. 1996). Mutation frequencies also increased in stationary phase compared with exponential phase (Supplemental Fig. S6C), suggesting that MMR proteins, including MutS, may become limiting in stationary phase, thus allowing accumulation of spontaneous mutations (Feng et al. 1996). Notably, the negative regulators of mutS discussed here, including Hfq, ArcZ, and SdsR, are all higher in stationary phase (Supplemental Figs. S3C, S8A).

Deletion of $\operatorname{arc} Z$ and $s d s R$ reduced mutagenesis (Supplemental Figs. S6D, S7A,B); such decreases could be attributed to derepression of mutS, and/or effects on other targets of these sRNAs. While deletion of $h f q$ also decreased mutagenesis (Fig. 6B; Supplemental Figs. S6E, S7A,B), it significantly affected cell growth (Supplemental Fig. S8C; Tsui et al. 1994) and had broad effects on gene expression (Guisbert et al. 2007). Deletion of rpoS decreased stress-induced mutagenesis only slightly more than deleting sdsR (Supplemental Fig. S7A,B).

We made use of the pathway-specific muts alleles (mutS ${ }^{\text {ArcZ }^{*}}, \mathrm{ARN}^{*}$, and mutS $^{\text {ArcZ }^{*}} \mathrm{ARN1}{ }^{*}$ ) in the native locus in the mutS leader to investigate the effects of mutS repression on mutagenesis. These mutants have important advantages over deletion of sRNAs or Hfq. They overcome pleiotropic effects from sRNA or $h f q$ deletions and did not interfere with bacterial growth (Supplemental Fig. S8C). In early stationary phase, cells carrying the mut $S^{\text {ArcZ* }^{*}}$ or ARN1* allele increased MutS levels by 1.8fold and 3.9-fold, respectively, and the double mutant mut ${ }^{\text {ArcZ* }}$ ARN1* increased MutS levels by 5.7-fold, comparable with that in the $h f q$ deletion mutant (Fig. 6A). These data suggest that these two pathways are responsible for Hfq-mediated mutS repression in stationary-phase cells. Notably, these mutS allele mutants did not change the levels of RpoS and Hfq proteins compared with wild type (Fig. 6A). When MutS levels were measured during bacterial growth, the $\mathrm{ARN1}^{*}, \mathrm{mutS}^{\mathrm{ArcZ}} \mathrm{ARN1}^{*}$, and $\Delta h f q$ mutants expressed high and comparable levels of MutS at all stages of growth, and the mutS ${ }^{\text {ArcZ }}{ }^{*}$ mutant had little or no effect (Supplemental Fig. S8D).

We then investigated how this increase in MutS in the leader mutants contributed to stationary-phase mutagenesis using the three assays for spontaneous and adaptive 
A

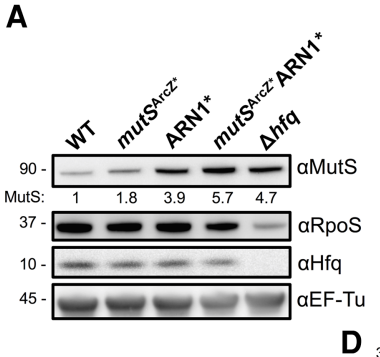

B

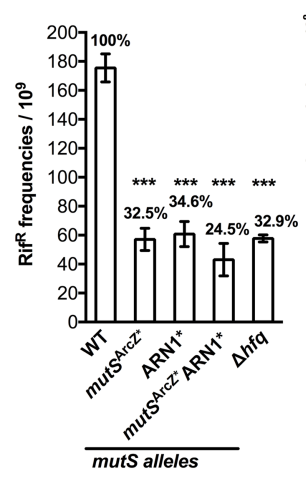

C
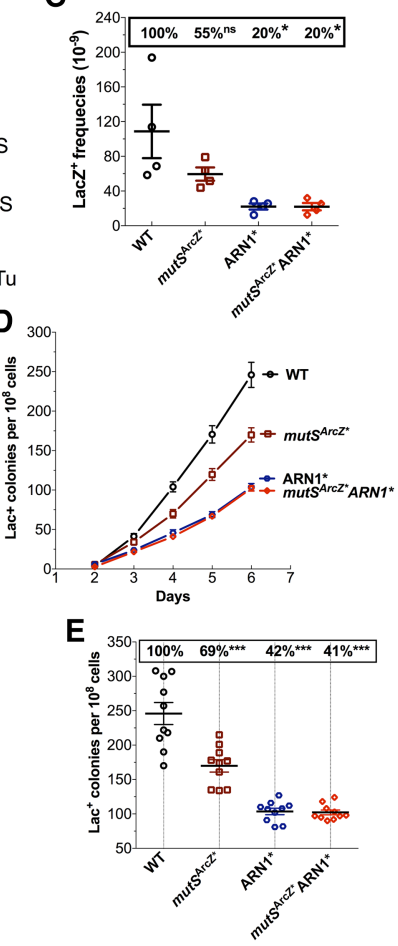

Figure 6. Hfq-mediated mutS repression contributes to stressinduced mutagenesis. (A) Bacterial cells (wild type: JC1081; $\Delta h f q$ : JC1090; mutS $^{\text {ArcZ* }}$ JC1083; ARN1*: JC1129; mutS $^{\text {ArcZ* }}$ ARN1*: JC1130) were grown in LB to stationary phase $\left(\mathrm{OD}_{600}\right.$ $\sim 2.5$; $\sim 6 \mathrm{~h}$ for JC1090 and $4 \mathrm{~h}$ for the other strains), and wholecell lysates from these cells were analyzed by Western blot using antibodies for MutS, RpoS, Hfq, and EF-Tu. EF-Tu served as a protein loading control. Representative blots from biological duplicates are shown with the relative MutS levels compared with wild type. $(B)$ The same set of strains was grown overnight (16 h) in $\mathrm{LB}$ at $37^{\circ} \mathrm{C}$ and tested for spontaneous resistance to $50 \mu \mathrm{g} /$ $\mathrm{mL}$ rifampicin. The frequencies for rifampicin resistance were determined by normalizing the number of colonies grown on selective LB rifampicin agar to that grown on nonselective LB agar. Data from three independent experiments were plotted as mean \pm SEM. (C) E. coli cells carrying a chromosomal lacZ ochre mutation (C70T) (wild type: JC1156; mutS $S^{\text {ArcZ* }}$ JC1157; ARN1*: JC1158; mutS $S^{\text {rrcZ }^{*}}$ ARN1*: JC1159) were grown in LB to stationary phase $\left(\mathrm{OD}_{600} \sim 3.5\right)$ and plated on M63 minimal lactose agar to measure $\mathrm{Lac}^{+}$reversion. The reversion frequencies were determined by normalizing the number of $\mathrm{Lac}^{+}$colonies grown on M63 minimal lactose agar to that of total viable cells grown on M63 minimal glucose agar. Data from four independent experiments were plotted as mean \pm SEM. $(D)$ Cells carrying a revertible lacIlac $Z$ frameshift mutation on the F episome as well as mutant alleles mutS $S^{\text {ArcZ }^{*}}$ or ARN1* in the mutS leader (wild type: JC1238; mut $S^{\text {ArcZ }^{*}}$ : JC1239; ARN1*: JC1240; mutS ${ }^{\text {ArcZ }}{ }^{*}$ ARN1*: JC1241) and isogenic scavenger allele variants (wild type: JC1234; mut $S^{\text {ArcZ* }}$ : JC1235; ARN1*: JC1236; mut $S^{\text {ArcZ }}{ }^{*}$ ARN1*: JC1237) were grown to saturation in $\mathrm{M} 9$ with $0.1 \%$ glycerol and assayed for adaptive mutation frequencies to $\mathrm{Lac}^{+}$as described in the Materials and Methods. (E) Dot plot of the total number of $\mathrm{Lac}^{+} \mathrm{col}-$ onies per $10^{8}$ viable cells on day 6 from the same set of strains as in $D$. Two independent isolates (five cultures each) for each construct were assayed, and data were plotted as mean + SEM. Unpaired Student's $t$-test (two-tail) was used to calculate statistical significance. $\left(^{*}\right) P<0.05 ;\left({ }^{* * *}\right) P<0.001$. mutagenesis discussed above. In stationary phase, all mutS mutants (mutS ${ }^{\text {ArcZ }}{ }^{*}, \mathrm{ARN}^{*}$, and mutS ${ }^{\mathrm{ArcZ}^{*}} \mathrm{ARN1}{ }^{*}$ ) showed significantly lower frequencies of rifampicin resistance compared with wild type, and the level was comparable with that in the $\Delta h f q$ strain (Fig. 6B). In the lac ochre reversion assay, mutS mutants ARN1* and mut $S^{\text {ArcZ }}{ }^{*}$ ARN1* showed significantly lower frequencies compared with wild type, comparable with that observed in the absence of Hfq, and the mut $S^{\text {ArcZ }}{ }^{*}$ mutant showed somewhat lower but statistically similar mutagenesis frequencies compared with wild type (Fig. 6C; Supplemental Fig. S6E). In the stress-induced $\mathrm{Lac}^{+}$reversion assay, ARN1* and mut $S^{\text {ArcZ }}$ ARN1* mutations significantly decreased the number of $\mathrm{Lac}^{+}$colonies at all time points, leading to a more than twofold decrease at day 6 ; the mut $S^{\text {ArcZ* }}$ mutation also significantly reduced mutation accumulation but less than the ARN1* strains (Fig. 6D, E). Thus, the mutagenesis levels, particularly in the Lac reversion assays, agreed well with MutS protein levels and suggest that MutS is limiting in wild-type cells, contributing to higher levels of mutagenesis.

$\mathrm{Lac}^{+}$revertant frequencies in the stress-induced mutagenesis assay on day 2 were generally assumed to have accumulated during cell growth and thus could be used as a measure of mutagenesis during growth. Deletion of mutS significantly increased mutation frequencies at day 2 , and deletion of $h f q, r p o S, \operatorname{arc} Z$, or $s d s R$ decreased mutation frequencies, although $\triangle r p o S$ was not statistically different from wild type (Supplemental Fig. S7C). The mutS leader mutants showed mutation frequencies similar to wildtype cells (Supplemental Fig. S7D). While the numbers of $\mathrm{Lac}^{+}$colonies were low at day 2, the results suggest that MutS may not be limiting during exponential growth. Collectively, these results demonstrate that mutS repression by Hfq and ArcZ sRNA contribute significantly to stress-induced mutagenesis in E. coli.

\section{Discussion}

The idea that mutation rates can be modulated in response to stress has been hotly debated over the last 30 years. Here we provide evidence that two distinct Hfq-dependent mechanisms control cellular MutS levels and thus contribute to E. coli stationary-phase mutagenesis.

\section{Noncanonical role of $\mathrm{Hf} q$ in direct translation inhibition} of mutS

Hfq is an RNA chaperone that binds to hundreds of mRNAs and dozens of sRNAs in E. coli (Zhang et al. 2013; Bilusic et al. 2014; Tree et al. 2014; Melamed et al. 2016). Our studies of Hfq-mediated muts repression have highlighted a noncanonical role of $\mathrm{Hfq}$ that can function in the absence of sRNA regulators.

Hfq strongly represses mutS-lacZ and endogenous mut $S$ expression in stationary-phase cells (Figs. 2,3; Tsui et al. 1997). This repression was largely dependent on an $(\mathrm{AAN})_{3}$ motif in the muts leader (ARN1) (Figs. 4, 6A). Two known sRNA regulators, ArcZ and SdsR, 
contributed modestly to mutS repression (Fig. 2). The Hfq proximal face is critical for binding all known Hfq-dependent sRNAs, and mutating Q8 on the proximal face to alanine leads to sRNA destabilization and loss of sRNAdependent regulation (Supplemental Figs. S2, S4D; Zhang et al. 2013; Schu et al. 2015) but did not eliminate Hfq repression of mutS (Fig. 3). An Hfq distal face mutation (Y25D), which impairs ARN-dependent target mRNA binding and regulation by Hfq (Ellis et al. 2015; Schu et al. 2015), mimicked the effect of deleting $h f q$ on mutS (Fig. 3). These in vivo data strongly suggest that sRNA binding plays a relatively minor role in mutS repression and rule out models in which we failed to find additional important sRNA regulators. Our in vivo results were confirmed and extended in vitro. Purified Hfq is sufficient to inhibit mutS translation in an ARN1-dependent manner (Fig. 5C). As the in vitro system is free of endonucleases, the primary role of Hfq can be attributed to translation inhibition rather than mRNA decay.

This is not the first report for Hfq regulation of gene expression on its own. Sonnleitner and Blasi (2014) demonstrated a role for $\mathrm{Hfq}$ in mediating carbon catabolite repression in Pseudomonas dependent on its distal face but independent of its proximal (sRNA-binding) face. Ellis et al. (2015) reported that Hfq represses IS10 transposition by binding to the RBS of transposase mRNA, also independent of the sRNA-binding face. Other studies show repression by Hfq in vivo and Hfq binding in vitro but have not examined the requirements for the sRNA-binding face of Hfq and therefore have not fully ruled out the participation of sRNAs /Vecerek et al. 2005; Salvail et al. 2013). In a variation on the ability of Hfq to repress, Hfq repressed $s d h C$ translation but was recruited to its binding site by a second interaction: pairing of Spot42 sRNA to the $s d h C 5^{\prime}$ UTR upstream of the RBS (Desnoyers and Masse 2012).
Hfq-mediated translational inhibition of mutS is somewhat different from what has been proposed in the cases discussed above. In previous reports, the Hfq-binding sites overlap the RBS sequence, and therefore Hfq directly competes with the ribosome $30 \mathrm{~S}$ subunit to inhibit translation. The Hfq-binding mutS ARN1 motif is $\sim 40 \mathrm{nt}$ upstream of the RBS sequence, beyond the distance for a direct interference with ribosome function (Huttenhofer and Noller 1994). The ARN1 motif itself did not affect translation in the absence of Hfq in vivo or in vitro (Figs. $4 \mathrm{~B}, 5 \mathrm{C})$. Therefore, unlike other examples of repression from a distant site, Hfq is not blocking a translational enhancer or ribosome standby site (Darfeuille et al. 2007; Yang et al. 2014). Rather, our structure-probing data (Fig. $5 \mathrm{D})$ support a model in which binding of the $(\mathrm{AAN})_{3}$ motif by the Hfq distal face rearranges the secondary structure of the muts leader, leading to occlusion of the RBS and/or the initiation codon for translation inhibition (Fig. 7A, bottom left; Supplemental Fig. S9). We suggest that Hfq binding releases the flexible binding of the $5^{\prime}$ end of the mutS to the translation initiation region (Fig. 5E), allowing a tighter inhibitory structure to form (Supplemental Fig. S9). Such a role of Hfq in restructuring mRNAs has been reported, although, in many cases, this restructured mRNA still requires sRNAs for regulation (Geissmann and Touati 2004; Soper et al. 2011; Ellis et al. 2015).

What makes the mutS ARN1 motif such an effective site for repression by Hfq? Recent studies (Robinson et al. 2014) suggest that the distal face of $E$. coli Hfq prefers AAN repeats, and the mutS ARN1 is a perfect AAN triplet sequence, tightly bound by Hfq with low nanomolar affinity; the upstream AU-rich Hfq-binding site (Fig. 5) may contribute to the cooperative binding and does contribute to Hfq repression (Supplemental Fig. S4). A systematic evolution of ligands by exponential enrichment (SELEX) screen for genomic sequences that tightly bind Hfq also

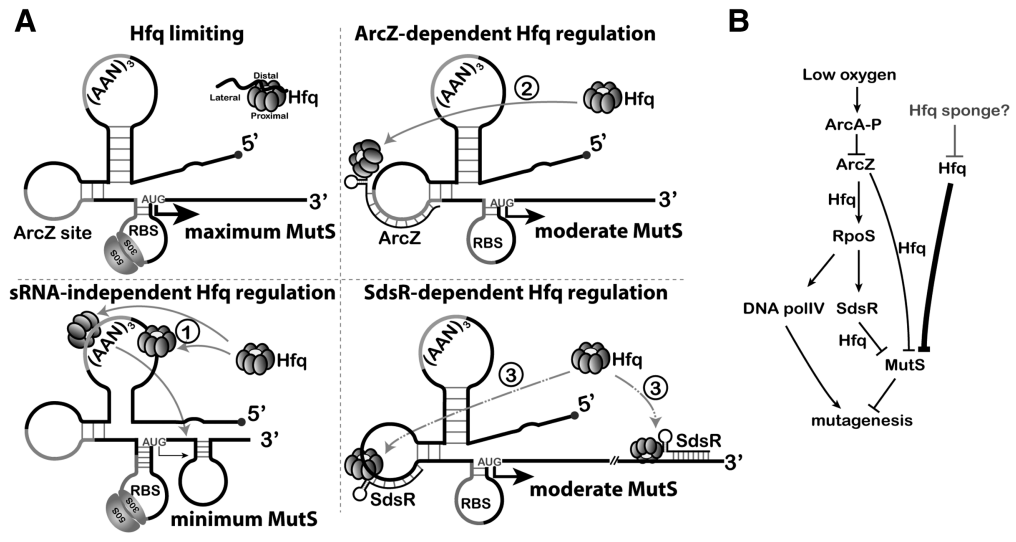

Figure 7. Models of Hfq-mediated mutS repression and underlying regulatory circuits of bacterial mutagenesis in E. coli. (A) The mutS leader folds into one strong $\left[(\mathrm{AAN})_{3}\right.$-containing] and two intermediate stem loops based on NUPACK prediction and our structure probing. (Top left panel) When Hfq is limiting (expression low or occupied by other RNAs), the muts leader adopts an open or flexible structure that allows ribosome loading and translation initiation from AUG, leading to maximal MutS production. When Hfq, ArcZ, or SdsR is abundant, Hfq can mediate repression of mutS by (1) binding to $(\mathrm{AAN})_{3}$ and upstream AU-rich regions through the distal face of Hfq, restructuring the muts leader, forming an inhibitory hairpin in the translation initiation region (Supplemental Fig. S9), and significantly downregulating mutS translation (bottom left panel); (2) binding to the mutS leader and ArcZ, leading to ArcZ pairing interfering with 30 S ribosome loading and dampening translation (top right panel); or (3) binding to the mutS leader at the $(\mathrm{AAN})_{3}$ site or elsewhere in the coding sequence and binding to SdsR, leading to SdsR-mediated translation inhibition or mutS mRNA decay (bottom right panel). These multiple layers of regulation allow potent MutS control with great flexibility. $(B)$ Hfq-mediated complex regulatory networks contribute to bacterial mutagenesis. Hfq can down-regulate MutS levels by direct translation inhibition (thick lines) as well as by promoting ArcZ or SdsR sRNA-mediated repression. Moreover, Hfq and ArcZ can increase the error-prone DNA polymerase IV through up-regulation of RpoS, leading to elevated mutagenesis in stationary phase or starvation conditions. See the text for references. 
identified a similar motif (Lorenz et al. 2010). The site protected by Hfq in the Pseudomonas amiE gene includes a perfect (AAN) ${ }_{5}$ repeat (Sonnleitner and Blasi 2014), although the IS10 site for Hfq repression of transposition is not quite as close to consensus (Ellis et al. 2015).

\section{Canonical role of $\mathrm{Hfq}$ in stabilizing ArcZ sRNA and assisting regulation}

Our work started by searching for sRNA regulators of mutS from a library of Hfq-dependent sRNAs. We identified two sRNA repressors: ArcZ and SdsR (Fig. 1). While this library was missing some of the recently identified sRNAs encoded at the $3^{\prime}$ ends of genes, a transcriptomewide mapping of sRNA-mRNA interactions after Hfq coimmunoprecipitation found only ArcZ and SdsR directly interacting with mutS mRNA on Hfq (Melamed et al. 2016), suggesting that there may not be any other sRNA regulators for mutS. SdsR was reported previously to base-pair with mutS deep in the coding sequence, based on in vitro studies (Gutierrez et al. 2013).

ArcZ is a conserved Hfq-dependent sRNA found in enterobacterial species (Papenfort et al. 2009; Mandin and Gottesman 2010). Unlike the sRNA-independent regulation of mutS, ArcZ repression requires the proximal and lateral faces of Hfq (Supplemental Fig. S2). ArcZ pairs with a sequence close to the mutS RBS, and this pairing likely blocks ribosome binding and translation initiation by a canonical repression mechanism. Deletion of $\operatorname{arc} Z$ increased MutS levels and decreased mutagenesis in stationary-phase cells (Fig. 2; Supplemental Fig. S6D), and mutation of the ArcZ-binding site in mutS had similar effects (Fig. 6). Therefore, ArcZ plays a significant role in mutS repression, although, under our test conditions, not as important as that of Hfq binding to ARN1.

The second sRNA, SdsR, is transcribed in stationary phase, dependent on the RpoS stationary phase/stress $\sigma$ factor (Frohlich et al. 2012). Gutierrez et al. (2013) reported that SdsR, which can be induced by low-dose ampicillin depending on RpoS, contributes to elevated mutagenesis presumably through down-regulating MutS levels. Indeed, overexpression of $S d s R$ in the $\Delta \operatorname{arc} Z \Delta s d s R$ strain significantly reduced both endogenous MutS levels as well as the mutS-lacZ fusion (Supplemental Fig. S1A,B), suggesting that a critical site for SdsR repression is within the muts leader and initial translated region.

\section{Integration and regulation of multiple roles of $\mathrm{Hfq}$ in mutS repression}

Our results revealed multiple modes of Hfq-mediated mutS repression (Fig. 7A): (1) direct inhibition of mutS translation by Hfq, dependent on the Hfq distal face and mutS ARN1 site; (2) ArcZ-mediated translation inhibition dependent on the proximal and lateral faces of $\mathrm{Hfq}$ and pairing between ArcZ and the mutS leader; and (3) SdsR-mediated mutS repression via the proximal and distal faces of Hfq and a site on the mutS leader and/or a previously reported site in the coding sequence. The first two pathways appear to act entirely independently, as our re- sults suggest that they use distinct mutS sites and Hfq faces. Mutations in ARN1 do not affect ArcZ-mediated repression, and mutations in mutS sites necessary for ArcZ repression do not disrupt direct repression by Hfq (Supplemental Fig. S4B; Fig. 5C). These independent mechanisms suggest that turning on either pathway can modulate cellular MutS levels. It is worth noting that the mutS leaders are not well conserved and that the ArcZ site as well as the ARN motif are present in only a limited number of bacterial species, including Enterobacter and Klebsiella; thus, these Hfq-mediated regulatory mechanisms may not be conserved in other organisms. Indeed, a search for SdsR-regulated mRNA targets in Salmonella enterica identified $>20$ targets but did not identify mutS (Frohlich et al. 2016). The SdsR-mediated mutS repression and Hfq direct inhibition are interconnected, as they both use the Hfq distal face as well as the mutS ARN1 site (Figs. 3, 4; Supplemental Fig. S4D,E); they are likely mutually exclusive.

Regulation by sRNAs generally correlates with conditions that induce sRNA expression. SdsR transcription is dependent on RpoS and thus should be expressed when the cells are experiencing an RpoS-inducing stress such as starvation, oxidative stress, or, as reported previously, sublethal doses of antibiotic (Gutierrez et al. 2013). ArcZ is negatively regulated by the two-component ArcB histidine kinase and ArcA response regulator, active anaerobically, and thus will be higher under aerobic conditions (Mandin and Gottesman 2010); it is highly expressed in stationary phase (Supplemental Fig. S3C). ArcZ positively regulates RpoS translation (Fig. 7B; Mandin and Gottesman 2010). Therefore, conditions that induce ArcZ will also induce SdsR via RpoS, and we noted lower levels of SdsR in cells deleted for arcZ (Supplemental Fig. S3B). These results suggest that under RpoS-inducing conditions, these sRNAs can collaborate to lower cellular levels of MutS.

Relatively little is known about how Hfq repression in the absence of sRNAs is regulated. Hfq levels are higher in stationary phase (Supplemental Fig. S8A), but mutating the ARN1 site is sufficient to increase MutS protein throughout growth, with the greatest effect seen in stationary phase (Supplemental Fig. S8D). Therefore, Hfq is presumably sufficient to repress MutS in both exponential growth and stationary phase. Possibly, Hfq-dependent repression simply provides a lower level of MutS protein, which can be further modulated in response to growth stresses. Even in the mutS ${ }^{\text {ArcZ }}{ }^{*}$ ARN1* mutant, MutS levels decreased in late stationary phase (Supplemental Fig. S8D), suggesting that yet other mechanisms may exist that keep MutS levels low in stationary phase.

\section{Roles of sRNAs and $\mathrm{Hfq}$ in regulation of bacterial mutagenesis}

Transient increases in mutation rates have been suggested to be a response to a variety of stresses (Galhardo et al. 2007), including stationary-phase stress. It has been suggested this is a bet-hedging strategy, allowing exploitation of mutations that may allow bacteria to deal with stress. 
One of the well-studied mutagenesis assays, referred to as adaptive or stress-induced mutagenesis, involves the accumulation of $\mathrm{Lac}^{+}$reversions of a frameshift mutation over many days of incubation (Foster 2007). Stress-induced mutagenesis depends on a variety of regulatory pathways, leading to up-regulation of error-prone polymerases (Foster 2007; Al Mamun et al. 2012). Less clear is to what extent these mutations depend as well on a regulated decline in MMR (Harris et al. 1999). Certainly, in either growing or stationary-phase cells, deletion of MMR components significantly increases the level of mutagenesis (Supplemental Figs. S6A,B, S7B). Experiments overexpressing MMR genes from plasmids and testing for stress-induced mutagenesis have led to conflicting results (Foster et al. 1995; Foster 1999; Harris et al. 1999; Gutierrez et al. 2013), although, overall, the results suggest either that MMR is limiting in stationary phase or, possibly, that overproducing the MMR proteins blocks processes needed for stressinduced mutagenesis. Our results demonstrate that MutS levels are controlled by multiple Hfq-dependent mechanisms, and this down-regulation contributes significantly to elevated mutagenesis in stationary phase (Fig. 6).

RpoS has been implicated in stress-induced mutagenesis (Bjedov et al. 2003; Al Mamun et al. 2012; Gutierrez et al. 2013). The two sRNAs found to down-regulate MutS are both intimately involved in the RpoS stress response. ArcZ is one of three sRNAs that positively induce rpoS translation (Mandin and Gottesman 2010), and the second sRNA, SdsR, is transcribed by RpoS (Fig. 7B; Frohlich et al. 2012). In our stress-induced mutagenesis assay, deletion of SdsR or RpoS led to similar declines in the mutagenesis frequencies (Supplemental Fig. S7), suggesting that an important role of RpoS is for SdsR synthesis. Given the well-known role of RpoS in stimulating synthesis of error-prone polymerases (Layton and Foster 2003), the SdsR deletion effect is somewhat surprising, but possibly these polymerases are not rate-limiting for mutagenesis unless MutS is also decreased. Presumably because both sRNAs are highly expressed as cells enter stationary phase (Supplemental Fig. S3C), the effect of these sRNAs on MutS levels is likely exerted in stationary-phase cells (Fig. 2C). Other conditions that lead to increased expression of either sRNA (for instance, any signal leading to induction of RpoS or conditions relieving the ArcB/ArcA repression of ArcZ [Mandin and Gottesman 2010]) are expected to lower MutS levels. Using a mutS allele that specifically blocks ArcZ pairing, we observed a moderate increase in endogenous MutS protein (Fig. 2) and a significant decrease in all of our mutagenesis assays (Fig. 6).

The stronger mode of mutS repression is via Hfq binding to the upstream region of the leader in the absence of sRNAs. While deletion of $h f q$ leads to a dramatic decrease in mutagenesis (Fig. 6; Supplemental Figs. S6E, S7), this mutation has pleiotropic effects on cells, decreasing synthesis of RpoS, leading to elevated synthesis and activity of RpoE and significantly slowing cell growth (Supplemental Fig. S8C; Tsui et al. 1994; Muffler et al. 1997; Guisbert et al. 2007). Importantly, we were able to specifically address the effect of Hfq on mutS by assays of cells carrying the mutS ${ }^{\mathrm{ARN1}^{*}}$ allele. In those cells, ArcZ is able to act, but the sRNA-independent repression of mutS is abrogated. The ARN1* mutation reduced mutagenesis significantly in all three mutagenesis assays, with the most significant effect (a fivefold reduction) seen in reversion of a lac ochre mutation (Fig. 6C). What was striking is that the effect of $\mathrm{ARN}^{*}$ on producing $\mathrm{Rif}^{\mathrm{R}}$ and lac ochre reversion mutants was very similar to that of deleting $h f q$ (35\% of wild type for $\mathrm{Rif}^{\mathrm{R}}$ in $\mathrm{ARN1}{ }^{*}, 33 \%$ in $\Delta h f q ; 20 \%$ of wild type for lac ochre reversion, and $24 \%$ for $\Delta h f q$ ) (Fig. 6B,C; Supplemental Fig. S6E), suggesting that MutS repression is likely the most important Hfq-dependent process in allowing mutagenesis. Unlike the effects of deleting $h f q$, the ARN1* cells grow normally but have elevated levels of MutS throughout growth (Supplemental Fig. S8D). What is less clear, however, is whether MutS is limiting for repair during exponential phase growth or whether Hfq repression leads only to less efficient MMR in stationary-phase cells. Our results support the latter, as increased MutS levels led to a decrease of mutation frequencies seen on day 6 but not day 2 (Fig. 6E; Supplemental Fig. S7D).

It is certainly likely that other sRNAs may also participate in regulation of mutagenesis and repair. OxyS, an Hfq-dependent sRNA induced after oxidative stress, was reported to reduce expression of RpoS and also reduced mutagenesis when overproduced (Altuvia et al. 1997). The decrease in RpoS is proposed to reflect titration of Hfq (Moon and Gottesman 2011), and certainly both less RpoS and less available Hfq could lead to higher levels of MutS and therefore less mutagenesis, consistent with a loss of the OxyS anti-mutator effect in muts mutants (Altuvia et al. 1997). Interestingly, the GcvB sRNA was shown recently to promote mutagenic break repair in $E$. coli, indirectly assisting the RpoS response by dampening membrane stress-induced $\sigma^{\mathrm{E}}$ competition (Barreto et al. 2016). We anticipate that the RNA chaperone Hfq as well as sRNAs play a significant role in balancing bacterial mutation rates, a role rarely appreciated for bacterial sRNAs.

\section{Materials and methods \\ Bacterial strains and culture conditions}

Bacterial strains used in this study were derivatives of E. coli K-12 MG1655. Strains and plasmids are listed in Supplemental Table S1, and strain constructions are described in the Supplemental Material. Primers, probes, and synthetic gene fragments (Integrated DNA Technologies) are listed in Supplemental Table S2. Transductions were carried out using phage P1vir as described by Miller (1972).

Bacterial strains were grown in Lennox broth (LB) medium with antibiotics where appropriate, unless otherwise indicated. M63 glucose or lactose agar (KD Medical) was used for strain construction and lac ochre reversion assay; M9 glycerol or lactose medium was used for adaptive mutagenesis experiments. When supplemented with glucose or lactose, a final concentration of $0.1 \%$ or $0.2 \%$ was used. Arabinose $(0.01 \%)$ was used for induction of $P_{B A D}$-mutS-lacZ expression, and a final concentration of 50 or $100 \mu \mathrm{M}$ IPTG was used for sRNA induction from plasmids. The concentrations of antibiotics used were $100 \mu \mathrm{g} / \mathrm{mL}$ ampicillin, $10 \mu \mathrm{g} / \mathrm{mL}$ chloramphenicol, $25 \mu \mathrm{g} / \mathrm{mL}$ kanamycin, $25 \mu \mathrm{g} / \mathrm{mL}$ zeocin, and $50 \mu \mathrm{g} / \mathrm{mL}$ rifampicin. 
Bacterial sRNA library screen

The sRNA plasmid library screen was carried out as described by Chen and Gottesman (2016) with the modification of $0.01 \%$ arabinose used for mutS-lac $Z$ induction. The $\beta$-galactosidase activities were determined as specific units calculated by $V_{\max }$ normalized to $\mathrm{OD}_{600}$ (Zhou and Gottesman 1998) and are significantly lower than Miller units. The effect from individual sRNA overexpression on mutS-lacZ expression was determined by comparison with the vector control (pBRplac).

\section{$\beta$-Galactosidase activity assay}

In general, bacterial cultures were either grown in LB for a given time $(6 \mathrm{~h})$ at $37^{\circ} \mathrm{C}$ or assayed at a similar optical density $\left(\mathrm{OD}_{600}\right.$ 3.0) for $\beta$-galactosidase assay as described by Miller (1972). Experiments were done in biological duplicates or triplicates, and mean and standard error are presented.

\section{Western blotting}

Western blotting was carried out using standard procedures as described in detail by Chen and Gottesman (2016). Antibody dilutions used were polyclonal rabbit anti-MutS antibodies (1:2500; Gene Check, Inc.), monoclonal mouse anti-EF-Tu antibody (1:10,000; LifeSpan BioSciences, Inc.), polyclonal rabbit antiRpoS antibodies (1:5000) (Battesti et al. 2015), polyclonal mouse anti-RpoD antibodies (1:5000), polyclonal rabbit anti-Hfq antibodies (1:2500) (Moon and Gottesman 2011), anti-rabbit AP-conjugated IgG (1:5000; Cell Signaling Technology), and anti-mouse AP-conjugated IgG (1:10,000; Santa Cruz Biotechnology). Western blot images were acquired by capturing the chemifluorescent signals using the Image Analyzer LAS-3000 (Fujifilm Life Science), ensuring signals in the linear range. The intensity of bands was quantified by using ImageStudio software and normalized to the signals of EF-Tu as a loading control.

\section{RNA EMSA}

Various mutS RNAs with $5^{\prime}$ end ${ }^{32} \mathrm{P}$ labeling were prepared as described in the Supplemental Material. Purified Hfq protein was a gift from Dr. Sarah Woodson's laboratory (Johns Hopkins University), and preparation was as described previously (Zhang et al. 2002). Binding reactions of Hfq with various mutS RNAs were carried out in a volume of $10 \mu \mathrm{L}$ as described (Lease and Woodson 2004) by combining $1 \mu \mathrm{L}$ of $10 \mathrm{nM}(10 \mathrm{fmol}){ }^{32} \mathrm{P}$ RNA, $2 \mu \mathrm{L}$ of Tris-EDTA buffer (KD Medical), $1 \mu \mathrm{L}$ of $1 \mathrm{mg} / \mathrm{mL}$ yeast tRNA (Ambion), $1.5 \mu \mathrm{L}$ of $20 \%$ glycerol, $2 \mu \mathrm{L}$ of $5 \times$ EMSA buffer (50 mM Tris- $\mathrm{HCl}$ at $\mathrm{pH} 8.0,250 \mathrm{mM} \mathrm{NaCl}, 250 \mathrm{mM} \mathrm{KCl}$ ), $2 \mu \mathrm{L}$ of various doses of Hfq protein or Hfq dilution buffer $(50$ $\mathrm{mM}$ Tris- $\mathrm{HCl}$ at $\mathrm{pH} 7.5,1 \mathrm{mM}$ EDTA, $250 \mathrm{mM} \mathrm{NH}_{4} \mathrm{Cl}, 10 \%$ glycerol), and $0.5 \mu \mathrm{L}$ of DEPC water. Reaction mixtures were incubated for $8 \mathrm{~min}$ at room temperature, and then samples were directly resolved on $6 \%(\mathrm{w} / \mathrm{v})$ native polyacrylamide gel in $0.5 \times \mathrm{TBE}$ buffer at $112 \mathrm{~V}$ for $1.2 \mathrm{~h}$ on ice. Resolved radiolabeled RNAs were exposed to film, and band intensities were quantified by using ImageStudio software (LI-COR, Inc.). Band intensity of free labeled RNA and ribonucleoprotein (RNP) was normalized to total signal in each lane and plotted using Prism 5 software (Graphpad) as the fraction of RNA bound $\left(f_{B}\right.$ mutS $)$ versus the concentration of $\mathrm{Hfq}_{6}$. Binding affinity $\left(K_{\mathrm{d}}\right)$ and Hill coefficient were calculated using Graphpad. Data from two independent experiments were plotted as mean \pm SEM.

\section{In vitro translation}

The RNA templates were in vitro transcribed using the MEGAshortscript $\mathrm{T} 7$ transcription kit (Ambion) according to the manufacturer's instructions from PCR products consisting of a T7 promoter sequence, mutS $5^{\prime}$ UTR plus the first 300 codons, and a C-terminal flag amplified by using primers JC108/JC116. The transcribed RNAs were treated with Turbo DNase (Ambion) and purified over a MEGAclear purification column (Ambion). The purified RNAs were checked on 6\% TBE-urea gel (Invitrogen) before $0.8 \mu \mathrm{g}(2.5 \mathrm{pmol})$ of RNA plus $2.5 \mathrm{pmol}$ of $\mathrm{Hfq}_{6}$ were used for in vitro translation using the standard protocol of PURExpress in vitro protein synthesis kit (Biolabs, Inc.). In vitro translated proteins were resolved on $4 \%-12 \%$ NuPAGE Bis-Tris gel (Invitrogen) and either stained with InstantBlue (Expedeon) for total proteins or probed with anti-Flag antibody (1:2500; Sigma, A9469 | for MutS'-Flag proteins.

\section{In vitro RNA structural probing and footprinting}

Hfq and mutS leader interactions were measured by RNA footprinting and structure probing using $\sim 0.3$ pmol of $5^{\prime}$ end ${ }^{32} \mathrm{P}$-radiolabeled mutS leader in $10-\mu \mathrm{L}$ reactions as described previously (Sharma et al. 2007). RNA was denatured for $1 \mathrm{~min}$ at $95^{\circ} \mathrm{C}$ and then chilled for $5 \mathrm{~min}$ on ice before mixing with $1 \mu \mathrm{g}$ of yeast RNA, $10 \times$ structure buffer [for Lead(II) and RNase T1] or 10× shortcut buffer $/ 10 \times \mathrm{MnCl}_{2}$ (for RNase III) and 3 pmol of $\mathrm{Hfq}_{6}$ or Hfq buffer. The Hfq-mutS leader-binding reactions were incubated for 10 min at room temperature before $2 \mu \mathrm{L}$ of $25 \mathrm{mM}$ fresh solution of lead(II) acetate (Fluka), $2 \mu \mathrm{L}$ of $0.01 \mathrm{U} / \mu \mathrm{L}$ RNase T1 (Ambion), or $1 \mu \mathrm{L}$ of $2 \mathrm{U} / \mu \mathrm{L}$ RNaseIII (Biolabs, Inc.) was added. Lead(II) and RNase $\mathrm{T} 1$ cleavage reactions were stopped after $4 \mathrm{~min}$ at $37^{\circ} \mathrm{C}$, and RNase III reaction was stopped after $5 \mathrm{~min}$ at $37^{\circ} \mathrm{C}$ by adding 20 $\mu \mathrm{L}$ of inactivation/precipitation buffer (RNase T1 kit, Ambion). Cleavage products were precipitated, washed, vacuum-dried, and resuspended in $10 \mu \mathrm{L}$ of loading buffer II (Ambion). RNase $\mathrm{T} 1$ ladders were generated using $0.3 \mathrm{pmol}$ of denatured RNA and incubation with $0.1 \mathrm{U}$ of RNase $\mathrm{T} 1$ for $5 \mathrm{~min}$ at $37^{\circ} \mathrm{C}$ in $1 \times$ sequencing buffer (Ambion). OH ladders were generated by incubating 0.3 pmol of RNA for $5 \mathrm{~min}$ at $95^{\circ} \mathrm{C}$ in the alkaline hydrolysis buffer (Ambion). Final samples were resolved on $8 \%$ polyacrylamide/7 $\mathrm{M}$ urea sequencing gel at $60 \mathrm{~W}$ for $1.2 \mathrm{~h}$ after heat denaturing for $5 \mathrm{~min}$ at $95^{\circ} \mathrm{C}$. Gels were vacuum-dried for $1 \mathrm{~h}$ at $80^{\circ} \mathrm{C}$ and exposed to a phosphorimager overnight. Scanned images were analyzed using ImageQuant software (GE Healthcare).

\section{Bacterial mutagenesis assays}

Wild type or mutS or $h f q$ mutants were grown in LB to late stationary phase for $16 \mathrm{~h}$ at $37^{\circ} \mathrm{C}$. Rifampicin-resistant clones were selected on $50 \mu \mathrm{g} / \mathrm{mL} \mathrm{LB}+$ Rif agar plates, and the number of total viable cells was determined by plating serial dilutions of cultures on nonselective LB agar plates. Colonies on selective or nonselective plates were counted after incubation for 48 and $24 \mathrm{~h}$, respectively, at $37^{\circ} \mathrm{C}$. The frequency of rifampicin resistance was calculated by dividing the mean of Rif $^{\mathrm{R}}$ cells by the total number of viable cells. For measuring mutation frequencies of reverting a lacZ ochre mutation (C70T) in various mutS allele or $\Delta h f q$ strains, bacterial overnight cultures were reinoculated $(1: 200)$ into fresh LB medium and grown for $6 \mathrm{~h}$ at $37^{\circ} \mathrm{C}$ before plating on M63 minimal lactose or glucose agar plates to measure $\mathrm{Lac}^{+}$ revertants or total viable cells, respectively. Plated cells were grown for $3 \mathrm{~d}$ at $37^{\circ} \mathrm{C}$ before counting, and $\mathrm{Lac}^{+}$reversion frequencies were calculated by dividing colony-forming units (CFUs) grown on M63 lactose with CFUs on M63 glucose agar (except for $\Delta h f q$ cells, which were plated on LB agar for counting total viable cells due to poor growth on minimal glucose agar). For each experiment, two strains for each construct were used, and biological duplicates or triplicates were assayed. Data are presented as mean plus standard error, and an unpaired Student's $t$-test was used to calculate statistical significance $\left(P<0.05\left[{ }^{*}\right]\right)$. 


\section{Adaptive mutagenesis assay}

An E. coli adaptive mutagenesis assay was carried out as described by Cairns and Foster (1991) using derivatives of strains provided by P. Foster. Strains with F' (1acI33-lacZ) (revertible) or $\mathrm{F}^{\prime} \Delta($ lacIZ) (scavenger) were grown in $1 \mathrm{~mL}$ of $\mathrm{M} 9$ minimal medium with $0.1 \%$ glycerol overnight at $37^{\circ} \mathrm{C}$. Overnight cultures were diluted $10^{5}$-fold into the same fresh medium, and five 1$\mathrm{mL}$ aliquots were grown to saturation in $24-48 \mathrm{~h}$. At the end of bacterial growth, the $\mathrm{OD}_{600}$ of cultures was measured, and $\sim 0.6 \times 10^{8}$ revertible cells ( $\sim 30 \mu \mathrm{L}$ of saturation cultures) were then mixed with $1.6 \times 10^{9}$ scavenger cells $(\sim 800 \mu \mathrm{L}$ of saturation cultures), pelleted, resuspended with $75 \mu \mathrm{L}$ of M9 salts, and spread on M9 plates containing $0.1 \%$ lactose. Meanwhile, the number of total revertible viable cells was numerated by plating on LB agar after serial dilution. Here, all scavenger cells were in the same genetic background as the revertible counterparts to eliminate viability issues, and these cells served to consume any contaminating carbon sources on the selecting agar, which otherwise might support the growth of revertible strains. M9 lactose plates were incubated for $7 \mathrm{~d}$ at $37^{\circ} \mathrm{C}$, and the number of revertants were counted from day 1 to day 6 . In this experimental setup, only 10-100 new $\mathrm{Lac}^{+}$colonies appeared each day from day 3 through day 6 . The post-plating mutation rates to $\mathrm{Lac}^{+}$ were calculated as the mean of $\mathrm{Lac}^{+}$colonies each day from days 2 to 6 per $10^{8}$ total viable cells. Two independent isolates for each construct were assayed, and data were plotted as mean plus standard error. The normal distribution of data sets was determined, and unpaired Student's $t$-test was used to calculate statistical significance $\left(P<0.05\left[{ }^{*}\right]\right.$ and $\left.P<0.01\left[{ }^{* *}\right]\right)$.

\section{Acknowledgments}

We thank S. Woodson for the gift of purified Hfq, S. Adhya for providing the lacZ ochre parental strain, and P. Foster for parental strains and protocols used for the adaptive mutagenesis. We thank G. Storz, M. Winkler, H.C. Tsui, T. Updegrove, P. Foster, and Gottesman laboratory members for comments on the manuscript. We also thank D. Lewis for technical guidance for the sequencing gel electrophoresis. Research in the Gottesman laboratory is supported by the Intramural Research Program of the National Institutes of Health, National Cancer Institute, Center for Cancer Research.

\section{References}

Al Mamun AA, Lombardo MJ, Shee C, Lisewski AM, Gonzalez C, Lin D, Nehring RB, Saint-Ruf C, Gibson JL, Frisch RL, et al. 2012. Identity and function of a large gene network underlying mutagenic repair of DNA breaks. Science 338: 1344-1348.

Altuvia S, Weinstein-Fischer D, Zhang A, Postow L, Storz G. 1997. A small, stable RNA induced by oxidative stress: role as a pleiotropic regulator and antimutator. Cell 90: 43-53.

Barreto B, Rogers E, Xia J, Frisch RL, Richters M, Fitzgerald DM, Rosenberg SM. 2016. The small RNA GcvB promotes mutagenic break repair by opposing the membrane stress response. I Bacteriol 198: 3296-3308.

Battesti A, Majdalani N, Gottesman S. 2015. Stress $\sigma$ factor RpoS degradation and translation are sensitive to the state of central metabolism. Proc Natl Acad Sci 112: 5159-5164.

Bilusic I, Popitsch N, Rescheneder P, Schroeder R, Lybecker M. 2014. Revisiting the coding potential of the E. coli genome through Hfq co-immunoprecipitation. RNA Biol 11: 641-654.
Bjedov I, Tenaillon O, Gerard B, Souza V, Denamur E, Radman M, Taddei F, Matic I. 2003. Stress-induced mutagenesis in bacteria. Science 300: 1404-1409.

Cairns J, Foster PL. 1991. Adaptive reversion of a frameshift mutation in Escherichia coli. Genetics 128: 695-701.

Chao Y, Li L, Girodat D, Forstner KU, Said N, Corcoran C, Smiga M, Papenfort K, Reinhardt R, Wieden HJ, et al. 2017. In vivo cleavage map illuminates the central role of RNase E in coding and non-coding RNA pathways. Mol Cell 65: 39-51.

Chen J, Gottesman S. 2016. Spot 42 sRNA regulates arabinose-inducible araBAD promoter activity by repressing synthesis of the high-affinity low-capacity arabinose transporter. I Bacteriol doi: 10.1128/JB.00691-16.

Darfeuille F, Unoson C, Vogel J, Wagner EG. 2007. An antisense RNA inhibits translation by competing with standby ribosomes. Mol Cell 26: 381-392.

Denamur E, Matic I. 2006. Evolution of mutation rates in bacteria. Mol Microbiol 60: 820-827.

Desnoyers G, Masse E. 2012. Noncanonical repression of translation initiation through small RNA recruitment of the RNA chaperone Hfq. Genes Dev 26: 726-739.

Ellis MJ, Trussler RS, Haniford DB. 2015. Hfq binds directly to the ribosome-binding site of IS10 transposase mRNA to inhibit translation. Mol Microbiol 96: 633-650.

Feng G, Tsui HC, Winkler ME. 1996. Depletion of the cellular amounts of the MutS and MutH methyl-directed mismatch repair proteins in stationary-phase Escherichia coli K-12 cells. I Bacteriol 178: 2388-2396.

Foster PL. 1999. Are adaptive mutations due to a decline in mismatch repair? The evidence is lacking. Mutat Res 436: 179-184.

Foster PL. 2007. Stress-induced mutagenesis in bacteria. Crit Rev Biochem Mol Biol 42: 373-397.

Foster PL, Gudmundsson G, Trimarchi JM, Cai H, Goodman MF. 1995. Proofreading-defective DNA polymerase II increases adaptive mutation in Escherichia coli. Proc Natl Acad Sci 92: 7951-7955.

Frohlich KS, Papenfort K, Berger AA, Vogel J. 2012. A conserved RpoS-dependent small RNA controls the synthesis of major porin OmpD. Nucleic Acids Res 40: 3623-3640.

Frohlich KS, Haneke K, Papenfort K, Vogel J. 2016. The target spectrum of SdsR small RNA in salmonella. Nucleic Acids Res 44: 10406-10422.

Fukui K. 2010. DNA mismatch repair in eukaryotes and bacteria. J Nucleic Acids 2010: 260512.

Galhardo RS, Hastings PJ, Rosenberg SM. 2007. Mutation as a stress response and the regulation of evolvability. Crit Rev Biochem Mol Biol 42: 399-435.

Geissmann TA, Touati D. 2004. Hfq, a new chaperoning role: binding to messenger RNA determines access for small RNA regulator. EMBO / 23: 396-405.

Grant CE, Bailey TL, Noble WS. 2011. FIMO: scanning for occurrences of a given motif. Bioinformatics 27: 1017-1018.

Guisbert E, Rhodius VA, Ahuja N, Witkin E, Gross CA. 2007. Hfq modulates the $\sigma^{\mathrm{E}}$-mediated envelope stress response and the $\sigma^{32}$-mediated cytoplasmic stress response in Escherichia coli. J Bacteriol 189: 1963-1973.

Gutierrez A, Laureti L, Crussard S, Abida H, Rodriguez-Rojas A, Blazquez J, Baharoglu Z, Mazel D, Darfeuille F, Vogel J, et al. 2013. $\beta$-Lactam antibiotics promote bacterial mutagenesis via an RpoS-mediated reduction in replication fidelity. Nat Commun 4: 1610.

Harris RS, Feng G, Ross KJ, Sidhu R, Thulin C, Longerich S, Szigety SK, Hastings PJ, Winkler ME, Rosenberg SM. 1999. 
Mismatch repair is diminished during stationary-phase mutation. Mutat Res 437: 51-60.

Holmqvist E, Wright PR, Li L, Bischler T, Barquist L, Reinhardt R, Backofen R, Vogel J. 2016. Global RNA recognition patterns of post-transcriptional regulators $\mathrm{Hfq}$ and CsrA revealed by UV crosslinking in vivo. EMBO J 35: 991-1011.

Huttenhofer A, Noller HF. 1994. Footprinting mRNA-ribosome complexes with chemical probes. EMBO J 13: 3892-3901.

Jensen PR, Hammer K. 1998. The sequence of spacers between the consensus sequences modulates the strength of prokaryotic promoters. Appl Environ Microbiol 64: 82-87.

Kery MB, Feldman M, Livny J, Tjaden B. 2014. TargetRNA2: identifying targets of small regulatory RNAs in bacteria. Nucleic Acids Res 42: W124-W129.

Kunkel TA, Erie DA. 2005. DNA mismatch repair. Annu Rev Biochem 74: 681-710.

Layton JC, Foster PL. 2003. Error-prone DNA polymerase IV is controlled by the stress-response $\sigma$ factor, RpoS, in Escherichia coli. Mol Microbiol 50: 549-561.

Lease RA, Woodson SA. 2004. Cycling of the Sm-like protein Hfq on the DsrA small regulatory RNA. J Mol Biol 344: 1211-1223.

LeClerc JE, Li B, Payne WL, Cebula TA. 1996. High mutation frequencies among Escherichia coli and Salmonella pathogens. Science 274: 1208-1211.

Li GM. 2008. Mechanisms and functions of DNA mismatch repair. Cell Res 18: 85-98.

Link TM, Valentin-Hansen P, Brennan RG. 2009. Structure of Escherichia coli Hfq bound to polyriboadenylate RNA. Proc Natl Acad Sci 106: 19292-19297.

Lorenz C, Gesell T, Zimmermann B, Schoeberl U, Bilusic I, Rajkowitsch L, Waldsich C, von Haeseler A, Schroeder R. 2010. Genomic SELEX for Hfq-binding RNAs identifies genomic aptamers predominantly in antisense transcripts. Nucleic Acids Res 38: 3794-3808.

Mandin P, Gottesman S. 2010. Integrating anaerobic/aerobic sensing and the general stress response through the ArcZ small RNA. EMBO I 29: 3094-3107.

Mandin P, Guillier M. 2013. Expanding control in bacteria: interplay between small RNAs and transcriptional regulators to control gene expression. Curr Opin Microbiol 16: 125-132.

Melamed S, Peer A, Faigenbaum-Romm R, Gatt YE, Reiss N, Bar A, Altuvia Y, Argaman L, Margalit H. 2016. Global mapping of small RNA-target interactions in bacteria. Mol Cell 63: 884-897.

Mika F, Hengge R. 2013. Small regulatory RNAs in the control of motility and biofilm formation in E. coli and Salmonella. Int $J$ Mol Sci 14: 4560-4579.

Miller JH. 1972. Experiments in molecular genetics. Cold Spring Harbor Laboratory, Cold Spring Harbor, NY.

Moon K, Gottesman S. 2011. Competition among Hfq-binding small RNAs in Escherichia coli. Mol Microbiol 82: 1545-1562.

Muffler A, Traulsen DD, Fischer D, Lange R, Hengge-Aronis R. 1997. The RNA-binding protein HF-I plays a global regulatory role which is largely, but not exclusively, due to its role in expression of the $\sigma^{\mathrm{S}}$ subunit of RNA polymerase in Escherichia coli. J Bacteriol 179: 297-300.

Otaka H, Ishikawa H, Morita T, Aiba H. 2011. PolyU tail of rhoindependent terminator of bacterial small RNAs is essential for Hfq action. Proc Natl Acad Sci 108: 13059-13064.

Panja S, Schu DI, Woodson SA. 2013. Conserved arginines on the rim of Hfq catalyze base pair formation and exchange. Nucleic Acids Res 41: 7536-7546.

Papenfort K, Said N, Welsink T, Lucchini S, Hinton JC, Vogel J. 2009. Specific and pleiotropic patterns of mRNA regulation by ArcZ, a conserved, Hfq-dependent small RNA. Mol Microbiol 74: 139-158.

Robinson KE, Orans J, Kovach AR, Link TM, Brennan RG. 2014. Mapping Hfq-RNA interaction surfaces using tryptophan fluorescence quenching. Nucleic Acids Res 42: 2736-2749.

Salvail H, Caron MP, Belanger J, Masse E. 2013. Antagonistic functions between the RNA chaperone Hfq and an sRNA regulate sensitivity to the antibiotic colicin. EMBO $I$ 32: 2764-2778.

Schu DJ, Zhang A, Gottesman S, Storz G. 2015. Alternative HfqsRNA interaction modes dictate alternative mRNA recognition. EMBO J 34: 2557-2573.

Schumacher MA, Pearson RF, Moller T, Valentin-Hansen P, Brennan RG. 2002. Structures of the pleiotropic translational regulator Hfq and an Hfq-RNA complex: a bacterial Sm-like protein. EMBO I 21: 3546-3556.

Sharma CM, Darfeuille F, Plantinga TH, Vogel J. 2007. A small RNA regulates multiple $\mathrm{ABC}$ transporter mRNAs by targeting C/A-rich elements inside and upstream of ribosome-binding sites. Genes Dev 21: 2804-2817.

Sobrero P, Valverde C. 2012. The bacterial protein Hfq: much more than a mere RNA-binding factor. Crit Rev Microbiol 38: 276-299.

Sonnleitner E, Blasi U. 2014. Regulation of Hfq by the RNA CrcZ in Pseudomonas aeruginosa carbon catabolite repression. PLoS Genet 10: e1004440.

Soper T, Mandin P, Majdalani N, Gottesman S, Woodson SA. 2010. Positive regulation by small RNAs and the role of Hfq. Proc Natl Acad Sci 107: 9602-9607.

Soper TJ, Doxzen K, Woodson SA. 2011. Major role for mRNA binding and restructuring in sRNA recruitment by Hfq. RNA 17: 1544-1550.

Sun X, Zhulin I, Wartell RM. 2002. Predicted structure and phyletic distribution of the RNA-binding protein Hfq. Nucleic Acids Res 30: 3662-3671.

Tree JJ, Granneman S, McAteer SP, Tollervey D, Gally DL. 2014. Identification of bacteriophage-encoded anti-sRNAs in pathogenic Escherichia coli. Mol Cell 55: 199-213.

Tsui HC, Leung HC, Winkler ME. 1994. Characterization of broadly pleiotropic phenotypes caused by an hfq insertion mutation in Escherichia coli K-12. Mol Microbiol 13: 35-49.

Tsui HC, Feng G, Winkler ME. 1997. Negative regulation of mutS and mutH repair gene expression by the Hfq and RpoS global regulators of Escherichia coli K-12. J Bacteriol 179: 7476-7487.

Updegrove TB, Zhang A, Storz G. 2016. Hfq: the flexible RNA matchmaker. Curr Opin Microbiol 30: 133-138.

Vecerek B, Moll I, Blasi U. 2005. Translational autocontrol of the Escherichia coli hfq RNA chaperone gene. RNA 11: 976-984.

Vogel J, Luisi BF. 2011. Hfq and its constellation of RNA. Nat Rev Microbiol 9: 578-589.

Yang Q, Figueroa-Bossi N, Bossi L. 2014. Translation enhancing ACA motifs and their silencing by a bacterial small regulatory RNA. PLoS Genet 10: e1004026.

Zadeh JN, Steenberg CD, Bois JS, Wolfe BR, Pierce MB, Khan AR, Dirks RM, Pierce NA. 2011. NUPACK: analysis and design of nucleic acid systems. J Comput Chem 32: 170-173.

Zhang A, Wassarman KM, Ortega J, Steven AC, Storz G. 2002. The Sm-like Hfq protein increases OxyS RNA interaction with target mRNAs. Mol Cell 9: 11-22.

Zhang A, Schu DJ, Tjaden BC, Storz G, Gottesman S. 2013. Mutations in interaction surfaces differentially impact $E$. coli $\mathrm{Hfq}$ association with small RNAs and their mRNA targets. J Mol Biol 425: 3678-3697.

Zhou Y, Gottesman S. 1998. Regulation of proteolysis of the stationary-phase $\sigma$ factor RpoS. J Bacteriol 180: 1154-1158. 


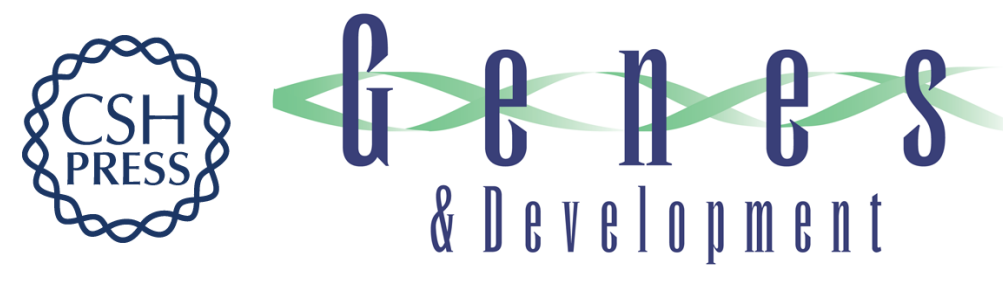

\section{Hfq links translation repression to stress-induced mutagenesis in $E$. coli}

Jiandong Chen and Susan Gottesman

Genes Dev. 2017, 31: originally published online August 9, 2017

Access the most recent version at doi:10.1101/gad.302547.117

\section{Supplemental http://genesdev.cshlp.org/content/suppl/2017/08/09/gad.302547.117.DC1 Material}

References This article cites 67 articles, 27 of which can be accessed free at: http://genesdev.cshlp.org/content/31/13/1382.full.html\#ref-list-1

License This is a work of the US Government. Email Alerting
Service

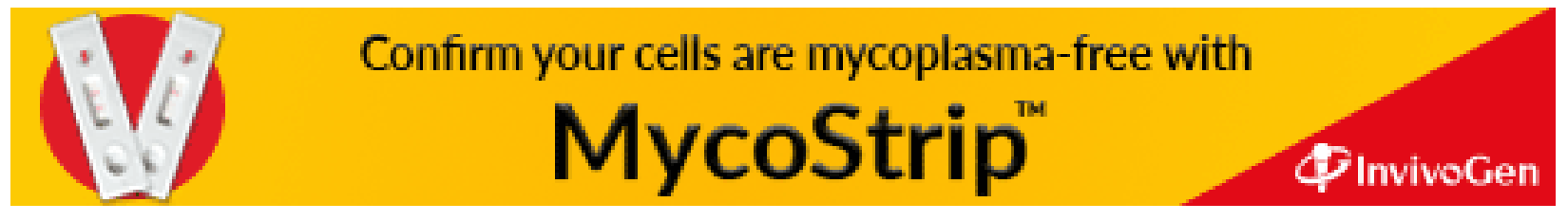

